\title{
Rainfall and water conditions in the region of the upper glacial in Europe
}

\author{
Zbigniew Szwejkowski, Ewa Dragańska, Iwona Cymes, Cristina Maria Timofte \\ University of Warmia and Mazury in Olsztyn, Department of Water Management Climatology and Environmental Man- \\ agement, Plac Łódzki 1/6, 10-718 Olsztyn,Poland, e-mail: szwzbig@uwm.edu.pl
}

\author{
Stanisław Suchecki \\ Institute of Meteorology Water Management - National Research Institute, Podleśna 61, 01-673 Warsaw, Poland
}

Ioan Craciun

Technical University “Gheorghe Asachi”, Bulevardul Profesor Dimitrie Mangeron 67, Iași 700050, Romania

\begin{abstract}
The article contains descriptive statistics on rainfall in an area of the southern part of the last ice age in Europe, and which now comprises the north-eastern part of the Poland. The study covers the period of 1981-2010. The sums of the annual and monthly precipitation were calculated, while years and months were evaluated in relation to the multi-annual and monthly averages. The frequency of daily precipitations was also determined. Values of SPI (Standarized Precipitation Index) were calculated together with the values of the Selianinov Index, which measures the environmental aspect of precipitation.

The average annual rainfall showed a spatial heterogeneity in the region from $700.1 \mathrm{~mm}$ (Elblag in the north-west) to $555.3 \mathrm{~mm}$ (Mława) in the east and south-east. The greatest precipitation occurred in the last decade of the analysed period of thirty years. However, statistical analysis shows no significant trend change in precipitation during this period. The number of years with sums of precipitation below $75 \%$ of the normal turned out to be very small. In the annual cycle, summer precipitation prevailed. On a monthly basis, the averages sometimes exceeded the multiyear averages by as much as $200 \%$, while extreme lows in monthly precipitation reached levels below $25 \%$ of the long-term averages. The calculated value of the SPI indicated that the most common drought conditions occurred in May and June; such droughts did not occur in April and July. The Selianinov Index indicates that the least favorable hydrothermal conditions occur in April and May, while the most favorable conditions occur in August and September.
\end{abstract}

Keywords: precipitation, drought, environment, upper glacial

Submitted 30 January 2015, revised 17 April 2015, accepted 3 October 2016

\section{Introduction}

Precipitation is the most dynamically changing element of the atmosphere, both in the weather and climatic context (Kuchar, Otop 2004; Orlińska-Woźniak et al. 2013). As it is one of the most important factors that determine the values of the environment, knowledge of the volume and distribution of precipitation is extremely important for the environment, agriculture, health protection, tourism, as well as in relation to industrial risk and individual hazards for people. The importance of precipitation makes research in this field important not only in its current state but also in the future, in the context of climate change. Climate change increases stresses on water resources in some watersheds, but decreases them in others (Arnell 2004). These findings, though interim and preliminary, highlight the growing challenges for water resource managers and highlight the need to explicitly address potential changes in climate and water use when developing future water management plans (Arnell 2004; Lehner et al. 2006).

North-eastern Poland, as a part of the area covered by the last ice age (the so called upper glacial), belongs to the complex of areas with a higher volume of precipitation than the mean for the whole country (Banaszkiewicz et al. 2004a, b, 2006; Suchecki et al. 2011) and, thus, the agricultural area includes a relatively large proportion of permanent grassland, and the scale of precipitation and its distribution does not present a substantial obstacle to the development of other business activities (Szwejkowski et al. 2002, 2005; Grabowska et al. 2004; Dragańska et al. 2005). The topography and the large number of natural water reservoirs, as a result of ice cap activity, neutralise the impact of sudden precipitation and spring thaws on the risk of flooding. However, floods do occur in Żuławy, located in the Vistula delta, although they are mainly caused by the mechanism of the so-called "cofka" ("outflow") 
into the Vistula and Nogat rivers from the Baltic body. Moreover, local flood risks occur in the main river beds of the Łyna, Drwęca and Pasłęka (ISOK 2014). Thus, this region stands out from other flood-threatened region sin Poland (Szalińska et al. 2014).

This paper presents the course of characteristics in the region over the last thirty years, including the first decade of the $21^{\text {st }}$ century; its objective is to search for an answer to the question of how the hydrological elements of the atmosphere will change in the next phase of progressive and global climatic changes.

\section{Methods}

The records of daily values of meteorological elements between 1981-2010 from the stations located in the area included in the analysis were the basis for the paper. The selected area is neither a separate geographical unit nor an administrative unit. However, it constitutes a part of Poland that is situated on the right side of the lower course of the Vistula River, with a conventional border on the south outlined roughly by Pojezierze Brodnickie, Wzniesienia Mławskie, Równina Kurpiowska, Pojezierze Ełckie and Równina Augustowska (Kondracki 1994). The selected area, which is conventionally referred to as North-Eastern Poland, was chosen - as an area converted by glaciers in the last ice age - and was based on the location of six meteorological stations administered by the Institute of Meteorology and Water Management: Elbląg, Kętrzyn, Mikołajki, Mława, Olsztyn and Suwałki. The data used in the present analysis were obtained at these stations.

All sets of data used in the analysis were checked for completeness and tested for homogeneity. The accuracy and reliability of the data were verified.

The analyses included daily precipitation sums. The basic values were processed with descriptive statistical methods $^{1}$ and compiled in appropriate tables. Precipitation was described with monthly and annual sums. Their time variability (tendency) was also determined. The relative value of precipitation was evaluated with the models developed by Przedpełska (1971) and expanded with authors assumptions.

An approximate index describing the fulfilment of environmental requirements for precipitation was established, based on known indices, such as periods without precipitation lasting longer than 10 days, the Standardized Precipitation Index $(S P I)$, the Selaninov Index, the intensity of precipitation, and the frequency of precipitation with thunder.

\footnotetext{
StatSoft, Inc. (2014). STATISTICA (data analysis software system), version 12, www.statsoft.com
}

\section{Results}

The annual precipitation sums in the selected areas were distinctly variable (Table 1). The highest annual means in the three-decade period at $700.1 \mathrm{~mm}$ were recorded in Elbląg, which is in the location closest to the Baltic Sea shore. The lowest precipitation was reported in Mława (on average $555.3 \mathrm{~mm}$ per year), which is situated in the southern part of the selected area and which directly neighbours part the Polish Lowland (a climatic area with the lowest precipitation in Poland). In the central and eastern part of the region, the average annual precipitation in 1981-2010 exceeded $600 \mathrm{~mm}$ and ranged from 601.1 in Kętrzyn to 637 in Olsztyn. In the vast majority of cases, the highest precipitation sums were recorded in the last decade of the examined period, i.e., from 2001-2010. In addition, there was a noticeable progression of precipitation (though this did not repeat at all measuring points) from one decade to another. By analysing the means for decades in different locations in the region it can be seen that they stayed within certain limits typical of a specific area. The course of isohyets reveals major regularity, which indicates the direction of spatial changes of the annual average precipitation sums. This direction signifies a decrease in precipitation sums from the north-western region towards the east and south-east (Fig. 1). The decade 1991-2000 is a departure from this rule.

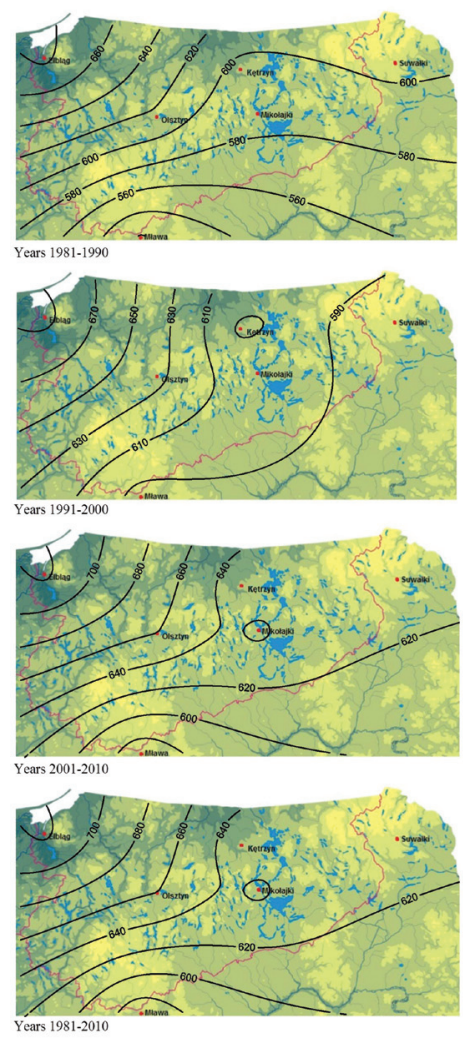

Fig. 1. Spatial distribution of annual precipitation sums in the years $1981-2010$ 
Table 1. Total monthly and yearly precipitation in ten-year intervals and average in the years 1981-2010

\begin{tabular}{|c|c|c|c|c|c|c|c|c|c|c|c|c|}
\hline $\begin{array}{c}\text { Weather stations/ } \\
\text { Months }\end{array}$ & 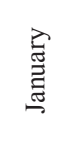 & $\begin{array}{l}\hat{E} \\
\text { 总 } \\
\text { 总 }\end{array}$ & 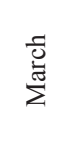 & $\overline{\bar{z}}$ & 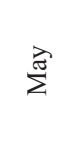 & 壭 & 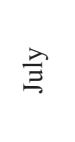 & $\begin{array}{l}\vec{w} \\
\underline{0} \\
\vec{E} \\
\vec{Z}\end{array}$ & 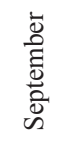 & $\begin{array}{l}\overline{0} \\
\text { 0. } \\
\dot{0}\end{array}$ & $\begin{array}{l}\overline{0} \\
\overline{0} \\
\overline{0} \\
\text { z }\end{array}$ & $\begin{array}{l}\dot{\Xi} \\
\text { हूँ } \\
\text { صू }\end{array}$ \\
\hline \multicolumn{13}{|c|}{$1981-1990$} \\
\hline Elbląg & 46,7 & 30,9 & 34,8 & 33,3 & 43,6 & 102,5 & 75,4 & 75,2 & 74,3 & 49,2 & 62,1 & 55,7 \\
\hline Kętrzyn & 37,8 & 20,1 & 33,1 & 29,0 & 53,3 & 89,8 & 80,1 & 65,5 & 57,3 & 36,8 & 47,2 & 42,5 \\
\hline Mikołajki & 33,8 & 21,5 & 29,8 & 26,5 & 58,2 & 100,7 & 67,1 & 78,3 & 53,5 & 33,6 & 46,3 & 38,6 \\
\hline Mława & 30,8 & 22,1 & 29,4 & 29,2 & 44,4 & 82,5 & 66,1 & 56,3 & 44,2 & 30,2 & 40,2 & 42,3 \\
\hline Olsztyn & 45,8 & 26,4 & 35,7 & 28,2 & 50,8 & 95,9 & 66,5 & 59,0 & 60,2 & 40,4 & 54,5 & 52,9 \\
\hline Suwałki & 41,5 & 21,9 & 32,6 & 28,9 & 59,3 & 91,0 & 77,8 & 63,1 & 59,1 & 41,2 & 48,1 & 41,8 \\
\hline \multicolumn{13}{|c|}{ 1991-2000 } \\
\hline Elbląg & 39,1 & 46,9 & 37,9 & 39,1 & 63,1 & 68,6 & 64,3 & 81,4 & 86,9 & 68,0 & 46,5 & 53,7 \\
\hline Kętrzyn & 33,0 & 33,5 & 38,7 & 45,8 & 55,6 & 78,2 & 62,5 & 60,5 & 57,4 & 41,3 & 43,7 & 35,8 \\
\hline Mikołajki & 29,0 & 35,0 & 43,1 & 44,9 & 55,4 & 70,9 & 72,1 & 69,0 & 61,0 & 45,2 & 42,7 & 38,7 \\
\hline Mława & 29,6 & 31,8 & 37,9 & 46,7 & 54,4 & 70,7 & 70,8 & 49,0 & 71,6 & 37,8 & 40,0 & 42,1 \\
\hline Olsztyn & 36,8 & 40,8 & 44,1 & 51,0 & 61,0 & 71,4 & 73,4 & 53,4 & 62,8 & 46,9 & 45,7 & 48,0 \\
\hline Suwałki & 31,9 & 35,5 & 40,8 & 45,0 & 45,4 & 63,4 & 73,1 & 56,9 & 53,6 & 45,3 & 49,7 & 45,0 \\
\hline \multicolumn{13}{|c|}{ 2001-2010 } \\
\hline Elbląg & 44,3 & 39,6 & 43,2 & 35,1 & 62,6 & 74,5 & 92,7 & 86,5 & 53,0 & 77,1 & 67,4 & 47,4 \\
\hline Kętrzyn & 32,3 & 31,9 & 33,9 & 26,7 & 58,1 & 71,5 & 80,7 & 95,8 & 54,2 & 56,4 & 49,9 & 33,8 \\
\hline Mikołajki & 36,4 & 31,8 & 31,2 & 26,2 & 65,2 & 77,9 & 79,9 & 104,0 & 56,2 & 57,0 & 43,6 & 34,8 \\
\hline Mława & 36,5 & 33,5 & 30,1 & 27,6 & 65,8 & 62,4 & 78,6 & 68,8 & 40,3 & 42,6 & 45,2 & 34,8 \\
\hline Olsztyn & 45,6 & 34,7 & 38,9 & 28,9 & 60,9 & 77,8 & 83,8 & 84,0 & 49,3 & 59,5 & 55,5 & 40,7 \\
\hline Suwałki & 41,8 & 35,7 & 34,7 & 23,2 & 64,6 & 66,8 & 91,4 & 87,6 & 49,6 & 57,5 & 40,8 & 35,1 \\
\hline \multicolumn{13}{|c|}{ 1981-2010 } \\
\hline Elbląg & 43,4 & 39,1 & 38,6 & 35,8 & 56,4 & 81,9 & 77,5 & 81,0 & 71,4 & 64,7 & 58,7 & 52,3 \\
\hline Kętrzyn & 34,3 & 28,5 & 35,2 & 33,8 & 55,7 & 79,8 & 74,4 & 73,9 & 56,3 & 44,8 & 46,9 & 37,4 \\
\hline Mikołajki & 33,1 & 29,4 & 34,7 & 32,5 & 59,6 & 83,1 & 73,0 & 83,8 & 56,9 & 45,3 & 44,2 & 37,4 \\
\hline Mława & 32,3 & 29,1 & 32,4 & 34,5 & 54,9 & 71,9 & 71,9 & 58,0 & 52,0 & 36,8 & 41,8 & 39,7 \\
\hline Olsztyn & 42,7 & 34,0 & 39,6 & 36,0 & 57,5 & 81,7 & 74,6 & 65,5 & 57,4 & 48,9 & 51,9 & 47,2 \\
\hline Suwałki & 38,4 & 31,0 & 36,0 & 32,4 & 56,4 & 73,7 & 80,8 & 69,2 & 54,1 & 48,0 & 46,2 & 40,7 \\
\hline
\end{tabular}

The area demonstrates a certain proportion of continental climate characteristics; these show typical seasonal differences in the annual distribution of precipitation. The highest precipitation occurred in summer, from June to August, whereas the lowest was from December to April. The highest monthly values in the three-decade period were recorded, with some exceptions, in June. In this month, the average precipitation in Elbląg was highest and reached $81.9 \mathrm{~mm}$. Relatively low means in August were recorded in Mława, Olsztyn, and Suwałki, amounting to $58.0,65.5$, and $69.2 \mathrm{~mm}$, respectively. The values recorded in July were intermediate. The lowest monthly precipitation sum, on average for the period 1981-2010, was recorded in February in Kętrzyn (only $28.5 \mathrm{~mm}$ ). For the other months, the mean for three decades ranged from 40 to $50 \mathrm{~mm}$ and the upper limit approximated $65 \mathrm{~mm}$ only in Elbląg. The minimum and maximum of monthly precipitation, considering the individual decades, were $20.1 \mathrm{~mm}$ (February 1981-2010 in Kętrzyn) and 104.0 (June 19812010 in Mikołajki), respectively. The precipitation sums decreased or were lower in June decade by decade, in relation to the first decade, in all locations, whilst in July and August the situation was the opposite: the highest values were always recorded in 2001-2010. The situation in winter and spring was not unambiguous, yet in the majority of cases the highest precipitation was recorded in the second
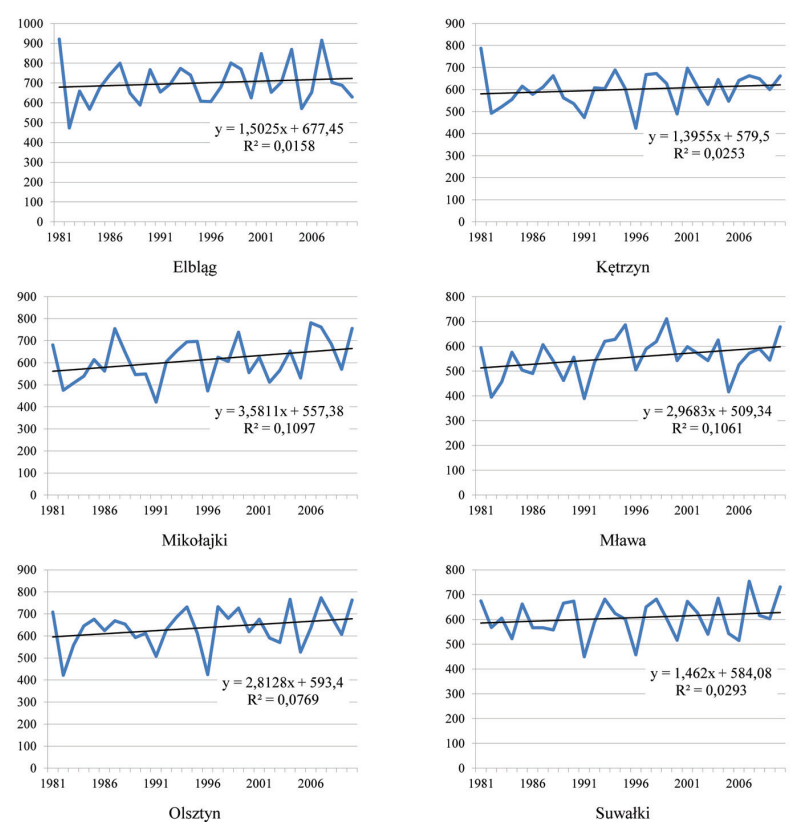

Fig. 2. Time trend of annual precipitation in the period 19812010 
Table 2. Time trends in precipitation totals in each month of the years 1981-2010

\begin{tabular}{|c|c|c|c|c|c|c|c|c|}
\hline Month & Time trend & $\mathrm{R}^{2}$ & Month & Time trend & $\mathrm{R}^{2}$ & Month & Time trend & $\mathrm{R}^{2}$ \\
\hline \multicolumn{3}{|c|}{ Elbląg } & \multicolumn{3}{|c|}{ Kętrzyn } & \multicolumn{3}{|c|}{ Mikołajki } \\
\hline January & $y=-0,1838 x+46,216$ & 0,007 & January & $y=-0,3508 x+39,781$ & 0,0264 & January & $y=0,0413 x+32,413$ & 0,0004 \\
\hline February & $y=0,4112 x+32,749$ & 0,0645 & February & $y=0,6207 x+18,865$ & 0,1767 & February & $y=0,5926 x+20,204$ & 0,2014 \\
\hline March & $y=0,4267 x+31,999$ & 0,0476 & March & $y=0,0197 x+34,894$ & 0,0001 & March & $y=0,1034 x+33,064$ & 0,0023 \\
\hline April & $y=0,0612 x+34,852$ & 0,0006 & April & $y=-0,2634 x+37,92$ & 0,0116 & April & $y=-0,1868 x+35,412$ & 0,0059 \\
\hline May & $y=0,9304 x+42,002$ & 0,1255 & May & $y=0,3924 x+49,591$ & 0,0337 & May & $y=0,6507 x+49,534$ & 0,0465 \\
\hline June & $y=-1,3971 x+103,5$ & 0,0919 & June & $y=-0,7249 x+91,039$ & 0,0414 & June & $y=-0,5385 x+91,493$ & 0,0131 \\
\hline July & $y=0,7891 x+65,229$ & 0,0346 & July & $y=-0,1003 x+75,982$ & 0,0006 & July & $y=0,5877 x+63,907$ & 0,0272 \\
\hline August & $y=0,7371 x+69,602$ & 0,0279 & August & $y=1,6972 x+47,637$ & 0,1528 & August & $y=1,588 x+59,136$ & 0,0748 \\
\hline September & $y=-1,5009 x+94,667$ & 0,0851 & September & $y=-0,52 x+64,35$ & 0,0261 & September & $y=-0,1751 x+59,601$ & 0,0019 \\
\hline October & $y=1,1992 x+46,149$ & 0,062 & October & $y=0,8411 x+31,793$ & 0,0621 & October & $y=1,0621 x+28,791$ & 0,0978 \\
\hline November & $y=0,3693 x+52,943$ & 0,0135 & November & $y=0,1295 x+44,923$ & 0,0034 & November & $y=-0,0494 x+44,989$ & 0,0006 \\
\hline December & $y=-0,3399 x+57,535$ & 0,0211 & December & $y=-0,3457 x+42,729$ & 0,0415 & December & $y=-0,095 x+38,835$ & 0,003 \\
\hline \multicolumn{3}{|c|}{ Mława } & \multicolumn{3}{|c|}{ Olsztyn } & \multicolumn{3}{|c|}{ Suwałki } \\
\hline January & $y=0,1776 x+29,531$ & 0,0067 & January & $y=-0,0784 x+43,946$ & 0,0008 & January & $y=-0,0876 x+39,762$ & 0,0011 \\
\hline February & $y=0,5967 x+19,878$ & 0,1633 & February & $y=0,4725 x+26,647$ & 0,1026 & February & $y=0,7061 x+20,066$ & 0,1544 \\
\hline March & $y=0,0894 x+31,054$ & 0,0029 & March & $y=0,2463 x+35,765$ & 0,0125 & March & $y=0,0806 x+34,744$ & 0,0017 \\
\hline April & $y=-0,0235 x+34,848$ & 0,0001 & April & $y=-0,0524 x+36,829$ & 0,0004 & April & $y=-0,2632 x+36,439$ & 0,0152 \\
\hline May & $y=1,2096 x+36,138$ & 0,1941 & May & $y=0,8885 x+43,776$ & 0,1007 & May & $\mathrm{y}=0,211 \mathrm{x}+53,126$ & 0,006 \\
\hline June & $y=-0,6068 x+81,291$ & 0,0236 & June & $y=-0,8035 x+94,148$ & 0,0397 & June & $y=-0,7235 x+84,941$ & 0,0436 \\
\hline July & $y=0,6347 x+62,016$ & 0,0298 & July & $y=1,0919 x+57,666$ & 0,0602 & July & $y=0,7363 x+69,34$ & 0,0283 \\
\hline August & $y=0,7107 x+47,008$ & 0,0532 & August & $y=1,4937 x+42,304$ & 0,1473 & August & $y=1,2601 x+49,635$ & 0,0808 \\
\hline September & $y=-0,4859 x+59,531$ & 0,0093 & September & $y=-1,0321 x+73,428$ & 0,059 & September & $y=-0,5507 x+62,639$ & 0,0239 \\
\hline October & $y=0,4327 x+30,133$ & 0,0231 & October & $y=0,7926 x+36,618$ & 0,0456 & October & $y=0,6691 x+37,633$ & 0,0398 \\
\hline November & $y=0,4656 x+34,569$ & 0,0406 & November & $y=0,2519 x+47,976$ & 0,0106 & November & $y=-0,2586 x+50,171$ & 0,0171 \\
\hline December & $y=-0,2325 x+43,34$ & 0,0126 & December & $y=-0,4581 x+54,294$ & 0,0331 & December & $y=-0,3177 x+45,58$ & 0,037 \\
\hline
\end{tabular}

decade (1991-2010). In autumn, a slightly higher proportion of decadal means was recorded in the last decade.

The sums of annual precipitation in the multi-annual period demonstrated a seasonal variability from year to year, with longer cycles being more rarely recorded. The course of a variability line for annual precipitation demonstrates that, in most cases, the highest precipitation in the annual scale was recorded at the beginning of the examined period (1981), and also in 2007. The driest years were: 1986, 1990, 2004, and 2006. Apart from this general statement, there were some differences at the individual stations. The statistical analysis did not demonstrate any significant tendency towards increasing or decreasing yearly sums of precipitation, putting aside the significance of the r-Pearson's Index.

In a monthly depiction, there are some examples of statistically significant tendencies indicating increases of precipitation in the multi-annual period. In such cases, however, as opposed to temperatures, there are fewer of them, and a certain degree of regional repeatability only concerns February (4 significant growth from 0.6 to $0.7 \mathrm{~mm} /$ year, out of 6 cases). Two locations demonstrated a significant increase in August, and one did so in May.

Based on the relative precipitation index according to Przedpełska (1971), it may be said that for most cases the annual precipitation sums remain within the range of normal conditions. The number of years with sums below
$75 \%$ of the multi-annual norms was found to be very small. Such situations were reported from 1 to 3 times in the years 1981-2010 (Table 3). Specifically, this concerns 1982 (depending on the location) and the years 1991 and 1996. The years with the highest precipitation sum include those in which the annual sums constituted not more than $131 \%$ of the multi-annual sum. In general, in the years with high precipitation, the norm was exceeded by 10 $20 \%$. On a monthly scale, the situation was more complicated; the extremely low precipitation as the means for the multi-annual period was lower than $25 \%$. The maximum exceedances of the norm in plus were by over $200 \%$. In general, low precipitation was typical of April, September, and October. There were, however, departures from that rule at individual meteorological stations. The first month of the vegetation period, mostly April, was very variable in precipitation and the year that stood out with a sum higher than $200 \%$ of the norm was 1994 in all locations; in April, however, there were also cases of very high precipitation, especially in 1999, when over $300 \%$ of the norm was recorded in Olsztyn and Mława. The middle periods of the year were driest in each decade and in all locations, but April 2009 was found to be the driest month in almost all locations, with sums ranging from $5 \%$ to $30 \%$ of the norm in Elbląg and Olsztyn, respectively. There were cases of serial springs that were dry or very dry in April, when the monthly sums did not exceed $75 \%$ of the multi-annual 
Table 3. Index of relative rainfall as percentages of the 1981-210 averages

\begin{tabular}{|c|c|c|c|c|c|c|c|c|c|c|c|c|c|}
\hline $\mathrm{yr} / \mathrm{mth}$ & I & II & III & IV & V & VI & VII & VIII & IX & $\mathrm{X}$ & XI & XII & Year \\
\hline 1981 & 87,6 & 94,3 & 95,8 & 93,3 & 63,4 & 180,6 & 195,1 & 181,4 & 63,0 & 123,7 & 188,4 & 110,6 & 131,3 \\
\hline 1982 & 127,1 & 35,0 & 36,5 & 73,5 & 62,9 & 162,9 & 69,2 & 35,9 & 17,5 & 46,5 & 53,4 & 74,4 & 67,6 \\
\hline 1983 & 172,0 & 72,1 & 155,9 & 167,6 & 118,6 & 18,4 & 28,1 & 87,3 & 122,5 & 47,1 & 171,1 & 82,1 & 94,0 \\
\hline 1984 & 93,2 & 55,7 & 95,8 & 23,2 & 60,3 & 190,2 & 77,8 & 16,8 & 169,0 & 74,5 & 22,8 & 26,6 & 81,0 \\
\hline 1985 & 81,6 & 82,6 & 81,1 & 66,2 & 80,8 & 156,1 & 89,2 & 115,3 & 139,6 & 26,9 & 48,4 & 133,7 & 96,2 \\
\hline 1986 & 188,6 & 45,2 & 62,9 & 179,3 & 121,2 & 102,0 & 90,8 & 123,2 & 106,0 & 59,6 & 75,9 & 137,6 & 105,7 \\
\hline 1987 & 116,4 & 116,3 & 96,9 & 75,7 & 86,3 & 148,7 & 106,0 & 136,4 & 104,5 & 36,3 & 164,3 & 154,8 & 114,0 \\
\hline 1988 & 94,1 & 115,8 & 98,9 & 49,7 & 80,3 & 101,2 & 157,5 & 68,5 & 75,8 & 19,8 & 102,3 & 141,8 & 92,6 \\
\hline 1989 & 63,4 & 80,0 & 67,9 & 114,0 & 10,6 & 103,0 & 27,6 & 86,0 & 36,3 & 199,1 & 109,3 & 119,6 & 84,0 \\
\hline 1990 & 53,5 & 93,0 & 108,5 & 87,2 & 87,6 & 88,7 & 131,9 & 77,9 & & 126,5 & 123,2 & 84,9 & 109,3 \\
\hline 1991 & 33,9 & 120,1 & 50,2 & 63,7 & 128,7 & 131,0 & 57,3 & 79,7 & 104,3 & 118,0 & 79,4 & 121,7 & 93,3 \\
\hline 1992 & 54,9 & 119,6 & 147,9 & 63,7 & 69,8 & 1,2 & 84,8 & 52,8 & & 109,4 & 103,5 & 96,4 & 99,3 \\
\hline 1993 & 144,6 & 164,4 & 112,7 & 40,2 & 128,0 & 143,6 & 102,4 & 124,3 & 179,3 & 15,0 & 48,6 & 101,2 & 110,4 \\
\hline 1994 & 144,4 & 86,9 & 160,6 & 151,7 & 66,1 & 56,8 & 19,9 & 82,1 & 143,8 & 193,7 & 74,1 & 171,6 & 105,6 \\
\hline 1995 & 127,7 & 69,3 & 90,6 & 147,2 & 77,5 & 65,6 & 28,1 & 148,1 & 96,4 & 51,1 & 118,6 & 52,6 & 86,8 \\
\hline 1996 & 38,3 & 111,4 & 22,8 & 26,3 & 176,3 & 64,4 & 145,1 & 86,1 & 120,0 & 86,3 & 43,8 & 48,6 & 86,4 \\
\hline 1997 & 38,3 & 116,3 & 76,7 & 141,9 & 134,9 & 34,6 & 104,6 & 33,8 & 143,0 & & 66,5 & 84,6 & 96,9 \\
\hline 1998 & 79,8 & 112,7 & 109,8 & 162,3 & 144,8 & 143,7 & 106,0 & 177,5 & 45,4 & 103,2 & 51,3 & 128,4 & 114,3 \\
\hline 1999 & 90,4 & 149,8 & 46,6 & & 156,1 & 117,3 & 80,2 & 122,2 & 27,3 & 139,3 & 104,3 & 100,3 & 109,9 \\
\hline 2000 & 149,2 & 147,0 & 163,9 & 55,0 & 36,3 & 80,0 & 101,1 & 97,4 & 56,7 & 19,8 & 101,6 & 122,4 & 89,2 \\
\hline 2001 & 92,0 & 105,3 & 140,6 & 179,1 & 60,1 & 44,0 & & 123,3 & 190,6 & 53,4 & 115,4 & 156,3 & 121,0 \\
\hline 2002 & 114,1 & 125,5 & 112,1 & 44,4 & 124,1 & 130,8 & 60,3 & 41,6 & 63,4 & & 59,8 & 36,5 & 93,2 \\
\hline 2003 & 97,3 & 66,5 & 36,0 & 174,3 & 72,1 & 106,0 & 152,1 & 48,1 & 76,2 & & 76,5 & 86,3 & 100,5 \\
\hline 2004 & 121,5 & 195,0 & 114,7 & 93,6 & 147,8 & 102,0 & 135,3 & 135,4 & 76,7 & 169,6 & 111,0 & 98,2 & 124,0 \\
\hline 2005 & 114,8 & 57,3 & 127,7 & 44,1 & 120,7 & 53,4 & 89,5 & 65,0 & 53,1 & 40,6 & 91,4 & 157,1 & 81,5 \\
\hline 2006 & 37,4 & 90,0 & 52,6 & & 107,8 & 73,7 & 46,6 & 146,0 & 58,8 & 50,4 & 193,5 & 71,4 & 93,2 \\
\hline 2007 & & 125,2 & 80,8 & 67,6 & 143,9 & 171,7 & 138,1 & 142,5 & 86,4 & 141,0 & 169,8 & 50,3 & 130,5 \\
\hline 2008 & 100,3 & 113,0 & 166,3 & 102,8 & 47,5 & 85,6 & 118,9 & 192,8 & 26,6 & 124,0 & 76,2 & 46,7 & 100,2 \\
\hline 2009 & 97,5 & 78,0 & & 5,0 & 145,2 & 107,1 & 167,3 & 31,0 & 36,7 & 168,8 & 64,3 & 70,6 & 98,2 \\
\hline 2010 & 44,7 & 56,7 & 81,6 & 43,6 & 140,4 & 35,7 & 84,9 & 141,7 & 73,8 & 26,9 & 191,4 & 132,8 & 89,8 \\
\hline
\end{tabular}

\begin{tabular}{|c|c|c|c|c|c|c|c|c|c|c|c|c|c|}
\hline & & & & & & & & & & & & & Kętrzy \\
\hline $\mathrm{yr} / \mathrm{mth}$ & I & II & III & IV & $\mathrm{V}$ & VI & VII & VIII & IX & $\mathrm{X}$ & XI & XII & Year \\
\hline 1981 & 46,9 & 83,9 & 96,0 & 49,7 & 138,8 & 166,7 & & 99,0 & 68,9 & 117,6 & & 133,3 & 131,0 \\
\hline 1982 & 150,5 & 28,1 & 52,6 & 77,1 & 105,3 & 85,6 & 91,5 & 108,5 & 36,6 & 31,0 & 65,2 & 128,7 & 82,0 \\
\hline 1983 & & 51,3 & & 136,2 & 91,6 & 38,7 & 65,8 & 47,1 & 77,5 & 56,2 & 75,6 & 89,6 & 86,8 \\
\hline 1984 & 138,6 & 56,2 & 99,4 & 73,3 & 65,7 & 104,6 & 135,8 & 62,5 & 171,8 & 65,4 & 55,8 & 33,7 & 92,4 \\
\hline 1985 & 62,3 & 68,5 & 76,1 & 111,7 & 97,4 & 127,1 & 101,6 & 93,0 & 167,0 & 80,1 & 67,3 & 129,2 & 102,4 \\
\hline 1986 & 161,9 & 25,3 & 36,9 & 148,4 & 81,7 & 92,0 & 81,7 & 135,9 & 101,1 & 85,7 & 60,9 & 131,1 & 96,3 \\
\hline 1987 & 68,1 & 120,4 & 40,1 & 62,9 & 131,7 & 118,3 & 85,5 & 109,0 & 122,9 & 66,3 & 109,1 & 148,8 & 101,6 \\
\hline 1988 & 90,6 & 58,3 & 77,6 & 44,6 & 116,2 & 156,8 & 168,5 & 94,5 & 68,4 & 27,0 & 181,3 & 139,4 & 110,3 \\
\hline 1989 & 91,7 & 88,8 & 89,8 & 125,9 & 57,5 & 116,4 & 43,1 & 66,7 & 71,1 & & 79,7 & 142,4 & 93,5 \\
\hline 1990 & 67,6 & 124,3 & 139,5 & 25,7 & 72,2 & 118,9 & 75,8 & 70,1 & 132,9 & 81,0 & 91,2 & 60,7 & 89,2 \\
\hline 1991 & 60,6 & 90,9 & 51,4 & 70,9 & 114,6 & 109,8 & 27,5 & 92,5 & 92,7 & 43,7 & 75,9 & 99,3 & 78,8 \\
\hline 1992 & 108,0 & 133,0 & 133,5 & & 76,5 & 32,7 & 48,5 & 28,3 & & 98,4 & 168,5 & 88,3 & 101,1 \\
\hline 1993 & 185,2 & 77,6 & 106,3 & 42,0 & 54,4 & 146,9 & 99,2 & 119,4 & 109,4 & 44,8 & 54,3 & 132,5 & 100,4 \\
\hline 1994 & 140,6 & 77,6 & & & 79,2 & 60,1 & 39,1 & 98,6 & 90,8 & 138,1 & 104,0 & 170,2 & 114,6 \\
\hline 1995 & 76,0 & 163,2 & 118,5 & 103,7 & 95,6 & 135,1 & 101,2 & 76,7 & 160,8 & 60,2 & 60,9 & 40,7 & 100,4 \\
\hline 1996 & 38,1 & 126,0 & 32,7 & 71,8 & 122,9 & 61,7 & 93,6 & 53,8 & 67,5 & 69,8 & 79,7 & 17,1 & 70,7 \\
\hline 1997 & 28,0 & 97,6 & 80,1 & & 93,6 & 93,4 & 118,2 & 90,9 & 118,8 & 174,4 & 147,9 & 97,9 & 111,1 \\
\hline 1998 & 155,5 & 147,4 & 86,4 & 129,7 & 112,3 & 186,3 & 105,5 & 108,6 & 37,3 & 101,7 & 75,4 & 84,3 & 112,0 \\
\hline 1999 & 43,7 & 161,5 & 52,8 & 174,4 & 172,3 & 108,8 & 88,9 & 65,7 & 40,7 & 185,1 & 84,8 & 121,0 & 104,3 \\
\hline 2000 & 125,8 & 100,7 & 155,1 & 47,0 & 78,0 & 44,9 & 118,4 & 84,0 & 68,8 & 4,0 & 79,5 & 107,0 & 81,4 \\
\hline 2001 & 57,4 & 67,0 & 106,5 & 175,5 & 61,8 & 120,0 & & 58,7 & & 77,8 & 86,1 & 80,5 & 116,0 \\
\hline 2002 & 143,8 & & 98,3 & 42,3 & 72,2 & 95,1 & 93,6 & 42,5 & 102,7 & 200,0 & 58,6 & 29,7 & 101,9 \\
\hline 2003 & 67,0 & 29,1 & 22,2 & 119,4 & 92,0 & 70,0 & 72,6 & 70,7 & 88,5 & 187,6 & 113,8 & 142,1 & 88,7 \\
\hline 2004 & 73,7 & 150,2 & 121,9 & 77,4 & 115,0 & 125,6 & 89,1 & 130,9 & 75,9 & 126,0 & 115,5 & 74,4 & 107,4 \\
\hline 2005 & 91,1 & 116,5 & 126,4 & 88,4 & 94,3 & 63,5 & 76,6 & 137,9 & 55,2 & 90,6 & 70,7 & 109,2 & 91,0 \\
\hline 2006 & 44,0 & 79,0 & 20,7 & 62,1 & 117,7 & 45,9 & 67,0 & & 150,3 & 45,3 & 162,4 & 95,0 & 106,7 \\
\hline 2007 & & 93,4 & 69,6 & 70,6 & 117,8 & 84,3 & 168,0 & 122,1 & 102,9 & 74,1 & 122,9 & 34,5 & 110,2 \\
\hline 2008 & 145,0 & 155,2 & 149,1 & 89,8 & 63,6 & 54,3 & 111,1 & 172,0 & 47,8 & 159,5 & 88,4 & 116,7 & 108,0 \\
\hline 2009 & 57,4 & 123,9 & 184,1 & 10,0 & 99,3 & 104,8 & 132,2 & 68,4 & 62,5 & 163,5 & 98,9 & 89,6 & 99,7 \\
\hline 2010 & 33,8 & 82,5 & 62,8 & 54,7 & & 131,9 & 55,6 & & 63,1 & 29,0 & 145,1 & 133,0 & 110,1 \\
\hline
\end{tabular}




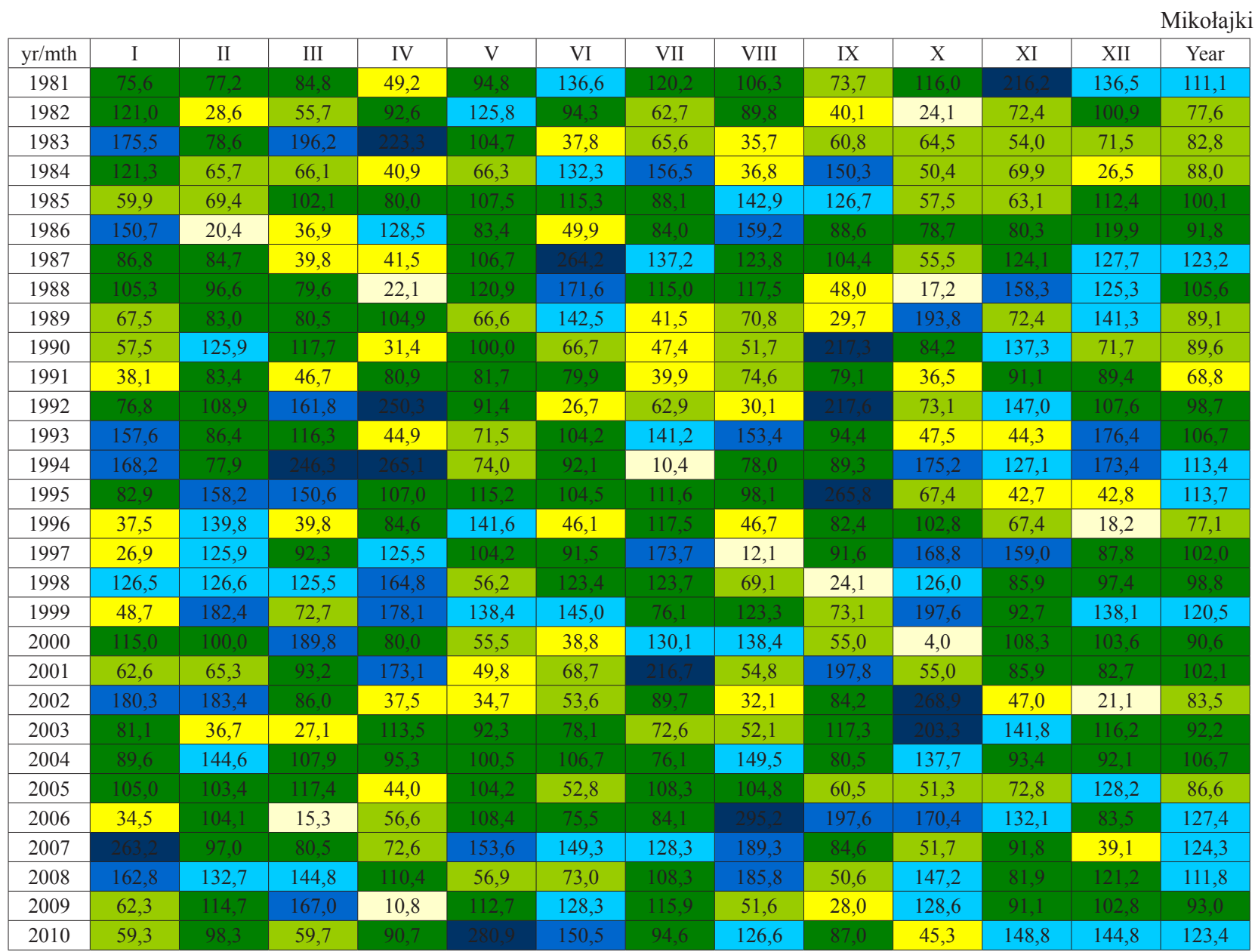

Mława

\begin{tabular}{|c|c|c|c|c|c|c|c|c|c|c|c|c|c|}
\hline $\mathrm{yr} / \mathrm{mth}$ & I & II & III & IV & $\mathrm{V}$ & VI & VII & VIII & IX & $X$ & XI & XII & Year \\
\hline 1981 & 93,9 & 70,0 & 108,5 & 40,0 & 59,6 & 140,8 & 96,2 & 173,4 & 46,9 & 174,5 & 131,1 & 116,8 & 106,8 \\
\hline 1982 & 91,7 & 35,4 & 46,5 & 73,9 & 93,8 & 107,0 & 64,3 & 82,0 & 27,5 & 58,6 & 55,3 & 82,0 & 71,0 \\
\hline 1983 & 143,7 & 102,7 & 178,2 & 130,5 & 100,8 & 39,4 & 49,4 & 88,6 & 43,7 & 71,7 & 66,5 & 73,2 & 82,0 \\
\hline 1984 & 105,0 & 72,4 & 94,3 & 56,3 & 75,4 & 124,2 & & 22,6 & & 42,3 & 89,5 & 22,6 & 103,7 \\
\hline 1985 & 54,5 & 70,4 & 98,3 & 122,7 & 103,5 & 110,6 & 65,7 & 100,1 & 97,1 & 41,5 & 70,4 & 136,9 & 90,7 \\
\hline 1986 & 156,4 & 60,8 & 52,4 & 66,4 & 121,5 & 48,0 & 92,0 & 109,4 & 76,9 & 72,5 & 68,2 & 140,9 & 88,3 \\
\hline 1987 & 93,2 & 74,8 & 39,8 & 83,8 & 82,0 & 162,6 & 117,5 & 95,8 & 106,3 & 92,6 & 170,4 & 124,8 & 109,1 \\
\hline 1988 & 128,9 & 90,6 & 93,1 & 12,5 & 81,3 & 100,2 & 117,9 & 159,4 & 42,3 & 10,9 & 126,4 & 166,6 & 97,5 \\
\hline 1989 & 39,0 & 82,7 & 89,7 & 158,9 & 54,7 & 162,5 & 28,8 & 51,4 & 16,0 & 159,1 & 55,5 & 135,4 & 83,2 \\
\hline 1990 & 48,9 & 99,2 & 103,9 & 101,8 & 36,8 & 152,3 & 71,3 & 88,2 & 182,7 & 96,1 & 128,7 & 64,9 & 100,0 \\
\hline 1991 & 66,0 & 74,2 & 56,4 & 76,6 & 72,5 & 118,0 & 50,5 & 42,6 & 48,1 & 47,0 & 106,7 & 73,5 & 70,1 \\
\hline 1992 & 47,4 & 81,4 & 142,4 & 134,6 & 59,0 & 49,7 & 91,0 & 40,3 & & 71,1 & 142,6 & 87,1 & 96,3 \\
\hline 1993 & 167,9 & 68,0 & 65,0 & 43,5 & 57,8 & 89,0 & 127,1 & 150,1 & & 33,7 & 53,4 & & 111,6 \\
\hline 1994 & 189,6 & 66,9 & 209,0 & & 89,8 & 29,6 & 20,7 & 92,4 & 118,5 & & 68,2 & 192,8 & 113,1 \\
\hline 1995 & 104,7 & 134,2 & 157,5 & 136,0 & 79,3 & 120,6 & 89,8 & 112,2 & 400,6 & 42,9 & 35,2 & 41,3 & 123,5 \\
\hline 1996 & 52,0 & 107,8 & 39,1 & 152,2 & 164,7 & 55,2 & 99,5 & 95,7 & 80,6 & 130,6 & 82,6 & 23,7 & 90,8 \\
\hline 1997 & 9,3 & 123,9 & 124,5 & 82,6 & 151,6 & 81,5 & 173,3 & 34,5 & 66,5 & 156,4 & 158,2 & 90,6 & 106,0 \\
\hline 1998 & 99,7 & 141,1 & 141,5 & 109,0 & 98,2 & 130,8 & 113,8 & 122,7 & 62,7 & 134,9 & 94,3 & 98,4 & 111,4 \\
\hline 1999 & 74,0 & 152,4 & 112,2 & 318.1 & 119,7 & & 73,8 & 75,0 & 43,7 & 130,8 & 90,5 & 107,2 & 128,0 \\
\hline 2000 & 104,7 & 140,8 & 121,1 & 74,8 & 99,3 & 54,9 & 146,0 & 78,8 & 87,1 & 17,1 & 124,9 & 136,6 & 97,7 \\
\hline 2001 & 43,7 & 81,0 & 72,7 & 144,1 & 89,5 & 91,3 & 142,5 & 124,9 & & 74,4 & 63,9 & 66,4 & 107,7 \\
\hline 2002 & 167,6 & & 127,3 & 32,2 & 37,2 & 78,7 & 103,7 & 83,6 & 62,7 & & 114,9 & 29,9 & 102,8 \\
\hline 2003 & 125,5 & 22,3 & 37,3 & 69,0 & 119,5 & 125,2 & 135,0 & 39,1 & 65,0 & 193,3 & 91,4 & 101,9 & 97,6 \\
\hline 2004 & 75,9 & 152,8 & 81,7 & 169,6 & 181,8 & 115,3 & 103,4 & 122,9 & 44,6 & 151,7 & 69,6 & 86,3 & 112,5 \\
\hline 2005 & 84,9 & 90,6 & 94,3 & 62,3 & 127,7 & 41,3 & 88,0 & 44,6 & 42,1 & 26,6 & 67,7 & 154,3 & 74,9 \\
\hline 2006 & 62,9 & 135,3 & 29,6 & 97,7 & 97,8 & 104,7 & 18,8 & 187,2 & 70,2 & 86,3 & 165,1 & 85,6 & 94,6 \\
\hline 2007 & & 113,0 & 96,8 & 56,3 & 86,5 & 64,5 & 148,6 & 139,8 & 69,2 & 41,0 & 67,5 & 65,9 & 102,9 \\
\hline 2008 & 130,7 & 130,5 & 160,6 & 118,3 & 109,0 & 33,1 & 135,3 & 179,1 & 51,7 & 141,4 & 51,7 & 79,3 & 106,2 \\
\hline 2009 & 57,3 & 84,5 & 142,4 & 8,7 & 99,5 & 119,2 & 139,6 & 85,8 & 32,5 & 149,8 & 119,7 & 99,2 & 98,0 \\
\hline 2010 & 69,4 & 81,4 & 83,5 & 41,8 & & 95,1 & 79,5 & 177,9 & 112,1 & 28,2 & & 107,5 & 122,1 \\
\hline
\end{tabular}




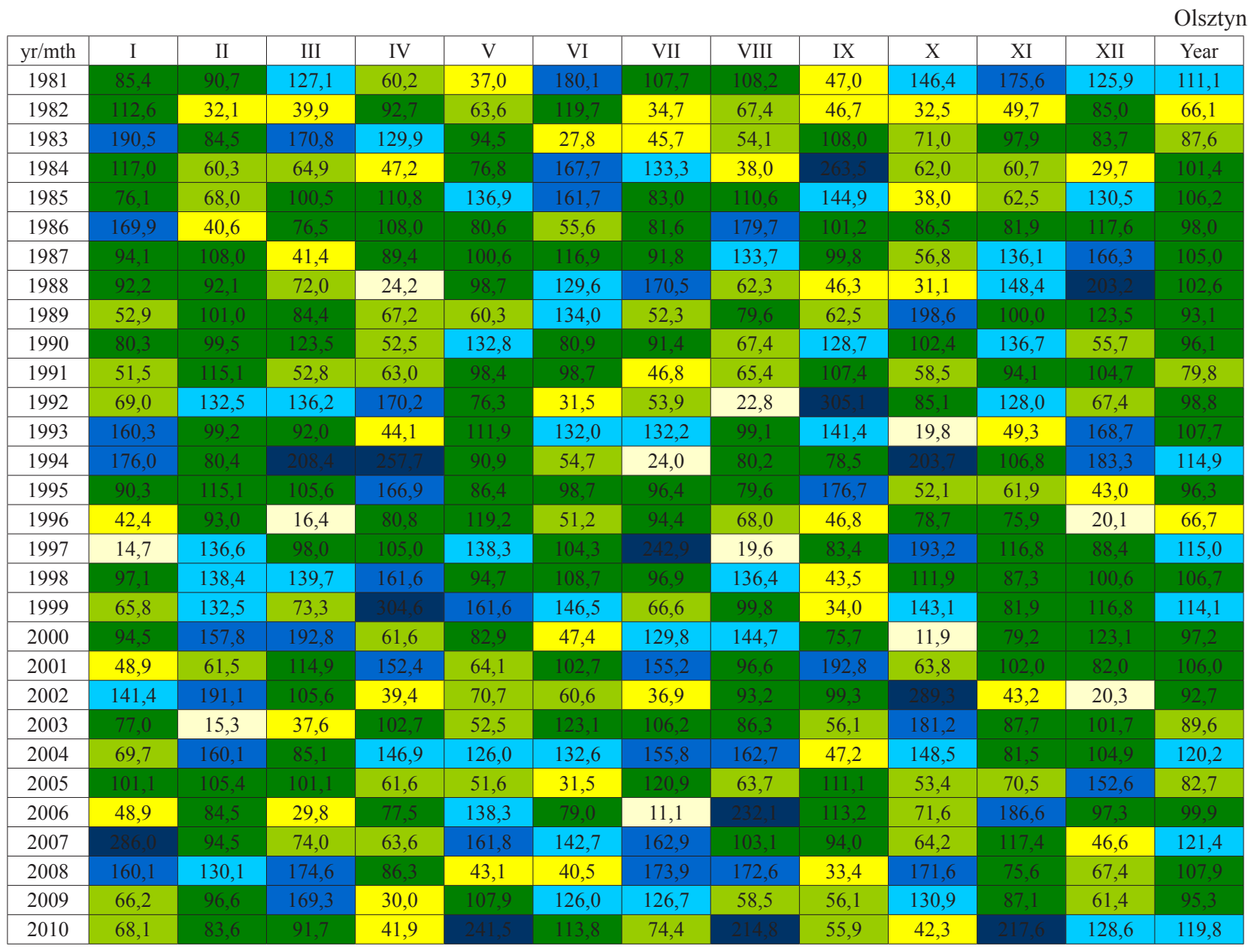

Olsztyn

\begin{tabular}{|c|c|c|c|c|c|c|c|c|c|c|c|c|c|}
\hline $\mathrm{yr} / \mathrm{mth}$ & I & II & III & IV & V & VI & VII & VIII & IX & $\mathrm{X}$ & XI & XII & Year \\
\hline 1981 & 85,4 & 90,7 & 127,1 & 60,2 & 37,0 & 180,1 & 107,7 & 108,2 & 47,0 & 146,4 & 175,6 & 125,9 & 111,1 \\
\hline 1982 & 112,6 & 32,1 & 39,9 & 92,7 & 63,6 & 119,7 & 34,7 & 67,4 & 46,7 & 32,5 & 49,7 & 85,0 & 66,1 \\
\hline 1983 & 190,5 & 84,5 & 170,8 & 129,9 & 94,5 & 27,8 & 45,7 & 54,1 & 108,0 & 71,0 & 97,9 & 83,7 & 87,6 \\
\hline 1984 & 117,0 & 60,3 & 64,9 & 47,2 & 76,8 & 167,7 & 133,3 & 38,0 & & 62,0 & 60,7 & 29,7 & 101,4 \\
\hline 1985 & 76,1 & 68,0 & 100,5 & 110,8 & 136,9 & 161,7 & 83,0 & 110,6 & 144,9 & 38,0 & 62,5 & 130,5 & 106,2 \\
\hline 1986 & 169,9 & 40,6 & 76,5 & 108,0 & 80,6 & 55,6 & 81,6 & 179,7 & 101,2 & 86,5 & 81,9 & 117,6 & 98,0 \\
\hline 1987 & 94,1 & 108,0 & 41,4 & 89,4 & 100,6 & 116,9 & 91,8 & 133,7 & 99,8 & 56,8 & 136,1 & 166,3 & 105,0 \\
\hline 1988 & 92,2 & 92,1 & 72,0 & 24,2 & 98,7 & 129,6 & 170,5 & 62,3 & 46,3 & 31,1 & 148,4 & & 102,6 \\
\hline 1989 & 52,9 & 101,0 & 84,4 & 67,2 & 60,3 & 134,0 & 52,3 & 79,6 & 62,5 & 198,6 & 100,0 & 123,5 & 93,1 \\
\hline 1990 & 80,3 & 99,5 & 123,5 & 52,5 & 132,8 & 80,9 & 91,4 & 67,4 & 128,7 & 102,4 & 136,7 & 55,7 & 96,1 \\
\hline 1991 & 51,5 & 115,1 & 52,8 & 63,0 & 98,4 & 98,7 & 46,8 & 65,4 & 107,4 & 58,5 & 94,1 & 104,7 & 79,8 \\
\hline 1992 & 69,0 & 132,5 & 136,2 & 170,2 & 76,3 & 31,5 & 53,9 & 22,8 & & 85,1 & 128,0 & 67,4 & 98,8 \\
\hline 1993 & 160,3 & 99,2 & 92,0 & 44,1 & 111,9 & 132,0 & 132,2 & 99,1 & 141,4 & 19,8 & 49,3 & 168,7 & 107,7 \\
\hline 1994 & 176,0 & 80,4 & 208,4 & & 90,9 & 54,7 & 24,0 & 80,2 & 78,5 & & 106,8 & 183,3 & 114,9 \\
\hline 1995 & 90,3 & 115,1 & 105,6 & 166,9 & 86,4 & 98,7 & 96,4 & 79,6 & 176,7 & 52,1 & 61,9 & 43,0 & 96,3 \\
\hline 1996 & 42,4 & 93,0 & 16,4 & 80,8 & 119,2 & 51,2 & 94,4 & 68,0 & 46,8 & 78,7 & 75,9 & 20,1 & 66,7 \\
\hline 1997 & 14,7 & 136,6 & 98,0 & 105,0 & 138,3 & 104,3 & & 19,6 & 83,4 & 193,2 & 116,8 & 88,4 & 115,0 \\
\hline 1998 & 97,1 & 138,4 & 139,7 & 161,6 & 94,7 & 108,7 & 96,9 & 136,4 & 43,5 & 111,9 & 87,3 & 100,6 & 106,7 \\
\hline 1999 & 65,8 & 132,5 & 73,3 & & 161,6 & 146,5 & 66,6 & 99,8 & 34,0 & 143,1 & 81,9 & 116,8 & 114,1 \\
\hline 2000 & 94,5 & 157,8 & 192,8 & 61,6 & 82,9 & 47,4 & 129,8 & 144,7 & 75,7 & 11,9 & 79,2 & 123,1 & 97,2 \\
\hline 2001 & 48,9 & 61,5 & 114,9 & 152,4 & 64,1 & 102,7 & 155,2 & 96,6 & 192,8 & 63,8 & 102,0 & 82,0 & 106,0 \\
\hline 2002 & 141,4 & 191,1 & 105,6 & 39,4 & 70,7 & 60,6 & 36,9 & 93,2 & 99,3 & & 43,2 & 20,3 & 92,7 \\
\hline 2003 & 77,0 & 15,3 & 37,6 & 102,7 & 52,5 & 123,1 & 106,2 & 86,3 & 56,1 & 181,2 & 87,7 & 101,7 & 89,6 \\
\hline 2004 & 69,7 & 160,1 & 85,1 & 146,9 & 126,0 & 132,6 & 155,8 & 162,7 & 47,2 & 148,5 & 81,5 & 104,9 & 120,2 \\
\hline 2005 & 101,1 & 105,4 & 101,1 & 61,6 & 51,6 & 31,5 & 120,9 & 63,7 & 111,1 & 53,4 & 70,5 & 152,6 & 82,7 \\
\hline 2006 & 48,9 & 84,5 & 29,8 & 77,5 & 138,3 & 79,0 & 11,1 & & 113,2 & 71,6 & 186,6 & 97,3 & 99,9 \\
\hline 2007 & & 94,5 & 74,0 & 63,6 & 161,8 & 142,7 & 162,9 & 103,1 & 94,0 & 64,2 & 117,4 & 46,6 & 121,4 \\
\hline 2008 & 160,1 & 130,1 & 174,6 & 86,3 & 43,1 & 40,5 & 173,9 & 172,6 & 33,4 & 171,6 & 75,6 & 67,4 & 107,9 \\
\hline 2009 & 66,2 & 96,6 & 169,3 & 30,0 & 107,9 & 126,0 & 126,7 & 58,5 & 56,1 & 130,9 & 87,1 & 61,4 & 95,3 \\
\hline 2010 & 68,1 & 83,6 & 91,7 & 41,9 & & 113,8 & 74,4 & & 55,9 & 42,3 & & 128,6 & 119,8 \\
\hline
\end{tabular}

\begin{tabular}{|c|c|c|c|c|c|c|c|c|c|c|c|c|c|}
\hline $\mathrm{yr} / \mathrm{mth}$ & I & II & III & IV & $\mathrm{V}$ & VI & VII & VIII & IX & $\mathrm{X}$ & XI & XII & Year \\
\hline 1981 & 62,8 & 70,3 & 85,3 & 55,3 & 104,8 & 134,8 & 138,0 & 110,2 & 77,1 & 103,7 & & 122,7 & 111,2 \\
\hline 1982 & 140,4 & 36,4 & 111,4 & 110,6 & 149,7 & 71,1 & 89,0 & 111,9 & 57,9 & 35,8 & 81,9 & 132,3 & 93,5 \\
\hline 1983 & & 86,1 & 159,2 & 143,7 & 159,4 & 113,7 & 56,3 & 26,9 & 55,4 & 82,5 & 79,3 & 68,9 & 99,7 \\
\hline 1984 & 136,4 & 94,5 & 27,8 & 55,9 & 112,2 & 102,4 & 86,4 & 16,9 & 180,2 & 73,7 & 73,9 & 61,7 & 86,1 \\
\hline 1985 & 66,7 & 50,0 & 83,6 & 115,6 & 71,3 & 116,5 & 114,4 & 189,0 & 134,9 & 89,4 & 78,4 & 127,2 & 109,0 \\
\hline 1986 & 114,6 & 22,9 & 47,2 & 103,2 & 121,6 & 93,0 & 76,4 & 97,7 & 145,3 & 60,2 & 109,8 & 98,1 & 93,3 \\
\hline 1987 & 45,0 & 66,8 & 50,3 & 48,2 & 92,0 & 177,5 & 83,1 & 102,8 & 117,2 & 30,4 & 109,2 & 110,9 & 93,3 \\
\hline 1988 & 105,2 & 80,0 & 114,5 & 32,4 & 91,7 & 86,4 & 152,2 & 100,8 & 63,6 & 15,8 & 101,2 & 108,5 & 91,9 \\
\hline 1989 & 60,9 & 76,7 & 109,5 & 196,5 & 92,7 & & 64,5 & 62,2 & 28,3 & & 63,7 & 141,9 & 109,7 \\
\hline 1990 & 80,5 & 121,6 & 116,1 & 30,9 & 55,9 & 135,9 & 102,4 & 93,4 & & 123,5 & 143,4 & 56,3 & 111,0 \\
\hline 1991 & 57,3 & 65,1 & 33,3 & 72,9 & 52,8 & 115,8 & 51,1 & 91,1 & 61,4 & 49,6 & 113,5 & 106,0 & 74,1 \\
\hline 1992 & 62,0 & 85,5 & 159,2 & & 59,8 & 27,7 & 36,5 & 55,8 & & 99,0 & 165,7 & 117,6 & 97,1 \\
\hline 1993 & 160,7 & 73,2 & 104,7 & 28,1 & 55,0 & 89,1 & 162,0 & & 100,9 & 69,0 & 49,6 & 181,0 & 112,3 \\
\hline 1994 & 162,0 & 84,8 & & 186,7 & 97,0 & 66,5 & 23,0 & 53,6 & 79,8 & 156,0 & 120,4 & 159,4 & 102,9 \\
\hline 1995 & 62,0 & 190,9 & 158,9 & 106,0 & 100,9 & 77,4 & 102,9 & 52,8 & 193,1 & 66,0 & 59,4 & 70,6 & 99,0 \\
\hline 1996 & 33,6 & 132,5 & 78,9 & & 90,4 & 51,4 & 78,1 & 39,8 & 56,4 & 95,6 & 72,8 & 35,9 & 75,5 \\
\hline 1997 & 31,5 & 106,1 & 55,6 & 96,4 & 184,9 & 113,4 & 141,5 & 18,7 & 109,2 & 156,2 & 144,3 & 94,9 & 107,2 \\
\hline 1998 & 97,9 & 152,9 & 137,8 & 183,3 & 60,6 & 126,0 & 124,0 & 128,5 & 73,2 & 116,7 & 76,9 & 100,1 & 112,4 \\
\hline 1999 & 60,9 & 142,5 & 42,0 & 167,5 & 78,7 & 149,9 & 53,9 & 97,9 & 54,2 & 130,0 & 101,6 & 153,0 & 99,5 \\
\hline 2000 & 103,4 & 110,6 & 145,9 & 100,7 & 23,9 & 43,1 & 131,9 & 83,3 & 52,9 & 6,5 & 171,8 & 89,0 & 85,0 \\
\hline 2001 & 71,9 & 86,7 & 143,4 & 87,8 & 121,8 & 79,9 & 136,1 & 97,7 & & 101,9 & 87,3 & 68,6 & 110,9 \\
\hline 2002 & 143,7 & & 101,7 & 57,8 & 100,2 & 100,8 & 95,4 & 22,0 & 40,3 & & 58,3 & 37,1 & 103,1 \\
\hline 2003 & 62,2 & 35,8 & 25,0 & 88,7 & 100,5 & 31,9 & 156,5 & 82,8 & 66,5 & 150,6 & 89,5 & 134,0 & 89,1 \\
\hline 2004 & 72,9 & 111,3 & 133,1 & 99,8 & 90,3 & 139,0 & 114,8 & 194,0 & 47,3 & 111,7 & 114,4 & 74,3 & 112,9 \\
\hline 2005 & 116,9 & 72,6 & 90,6 & 72,0 & 130,5 & 94,0 & 57,7 & 149,6 & 74,3 & 52,1 & 46,6 & 99,1 & 89,5 \\
\hline 2006 & 32,3 & 102,5 & 26,9 & 56,2 & 77,8 & 37,8 & 28,4 & & 134,4 & 111,9 & 75,4 & 98,9 & 85,0 \\
\hline 2007 & & 91,3 & 91,7 & 60,3 & 137,1 & 83,7 & & 89,2 & 141,0 & 64,0 & 77,8 & 45,5 & 124,2 \\
\hline 2008 & 167,2 & 173,8 & 164,2 & 69,8 & 37,1 & 79,5 & 80,1 & 150,7 & 68,2 & 112,9 & 75,4 & 102,8 & 101,5 \\
\hline 2009 & 80,7 & 93,8 & 103,4 & 17,3 & 133,3 & 131,7 & 114,7 & 73,6 & 36,0 & 146,4 & 126,1 & 87,6 & 99,3 \\
\hline 2010 & 61,7 & 103,5 & 83,1 & 106,0 & & 127,4 & 97,0 & 193,7 & 93,9 & 51,2 & 132,6 & 115,8 & 120,6 \\
\hline
\end{tabular}


Description for Table 3: percentage (\%) of rainfall of a month in relation to the multi-year average 1981-2010

$\begin{array}{ll}\text { months } & \text { years } \\ >200 & >155 \\ 150,1-200 & 135,1-155 \\ 125,1-150 & 110,1-135 \\ 75,1-125 & 90,1-110 \\ 50,1-75 & 75,1-90 \\ 25,1-50 & 50,1-75 \\ <25 & <50\end{array}$

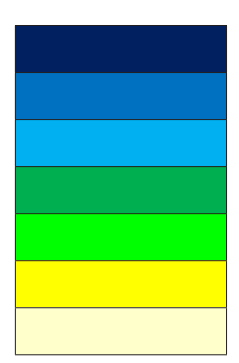

norm. The longest period with such characteristics was recorded in 2005-2009 (5 years) in Suwałki. A 4-year series was reported in Olsztyn in 1988-1991. There were 3-year series in other locations, especially at the beginning of the second decade of the analysed three-decade period. The periods of drought with less than $50 \%$ of the multi-annual precipitation sum for May were less frequent than in April. In some cases, however, the sums lower than $75 \%$ of the norm which occurred in a series of years with an uneven distribution in time presented a problem - for instance, for agricultural production. June was drier than May in the analysed three-decade period. In July and August, the situation was markedly diversified. In the subsequent years following 1991, precipitation was below the norm by at most $30 \%$, yet even in those months with the highest multi-annual averages, there were cases of the norm being exceeded by $100 \%$ or more. Apart from 2001, such a situation did not occur at the same time in all locations; on the contrary, such cases were recorded for each situation in a different year. September and October were most variable in the precipitation sum year-to-year when the norm was exceeded by up to $200 \%$, alternating with situations when the precipitation level was a few per cent of the norm. In 1992, in all locations, the norm for September was exceeded twice. In September 1995, at the station located in Mława, rain meters showed a record-high overrun of the multi-annual norm, amounting to $400.6 \%$. The winter period also demonstrated a varied picture of the situation. Precipitation in December (predominantly snow) had decreasing sums in the three-decade period. As a result, a sort of regularity appears for December, namely, the relative values are higher in the first ten-day period than in the second and third ones of this month, especially in 19851990. In January, a clear overrun of the multi-annual norm was recorded in 1983 and in 2007. A series of much lower precipitation occurred in January in 1996-1997. The precipitation that was higher than the norm in February was typical of 1995-2000.

A precipitation sum does not always correctly indicate a level of water in the environment that signifies deficits or surpluses in relation to needs, especially for cultivated plants. Longer periods without precipitation are most troublesome for the biosphere, especially at higher positive temperatures. Therefore, included in Table 4 are the values indicating the number of incidents and the duration of periods without precipitation during plant vegetation from April to September. This data shows that the frequency of periods lasting longer than 10 days was from 50 to 59 of such incidents in the three decade-long periods (19812010), with the highest number recorded in Kętrzyn and Mikołajki (the central part of the region) and the lowest recorded in Elbląg. In this case, the average annual frequency of such periods approximated two. The variability indices for this characteristic of the climate over the threedecade period, were 1.53 per year at their maximum (Olsztyn, Mława), but they were generally lower, at 1.16-1.28 per year. There was no statistical tendency for a change of the situation over time, which is demonstrated by

Table 4. Periods of days without precipitation

\begin{tabular}{|c|c|c|c|c|c|c|}
\hline Parameter & 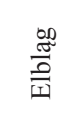 & 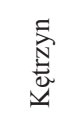 & $\begin{array}{l}\frac{\vec{y}}{\pi} \\
\frac{\pi}{0} \\
\frac{y}{2}\end{array}$ & $\sum_{\Sigma}^{\pi}$ & 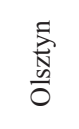 & 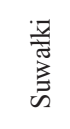 \\
\hline Number of periods without precipitation longer than 10 days & 50 & 58 & 59 & 55 & 56 & 52 \\
\hline Mean years of periods without precipitation & 1,67 & 1,93 & 1,97 & 1,83 & 1,87 & 1,73 \\
\hline Coefficient of variability & 1,18 & 1,28 & 1,16 & 1,53 & 1,53 & 1,17 \\
\hline $\mathrm{R}^{2}$ of the trend & 0,01 & 0,01 & 0,08 & 0,06 & 0,02 & 0,07 \\
\hline Mean days of periods without precipitation & 14,68 & 14,17 & 14,00 & 14,47 & 14,36 & 15,31 \\
\hline Longest period without precipitation in days & 31 & 34 & 39 & 31 & 25 & 39 \\
\hline $\begin{array}{l}\text { Number of periods with total sum of precipitation less than } 40 \mathrm{~mm} \\
\text { during sixty days, following periods without precipitation }\end{array}$ & 8 & 14 & 9 & 12 & 8 & 6 \\
\hline
\end{tabular}


exceptionally low $\mathrm{R}^{2}$ values. The periods lasting for over 10 days with no precipitation were evaluated, and the longest average period without precipitation in the region over the three-decade period was recorded in Suwałki 15.31 days, while the shortest was in Mikołajki (14 days). The absolutely longest period, lasting 39 days, was also recorded in Suwałki and Mława. Periods without precipitation were preceded by precipitations that were higher. When a period without precipitation was preceded by a 60-day precipitation sum lower than $40 \mathrm{~mm}$, the situation was then perceived as very bad. The worst situation, when 14 of the 58 dry periods were preceded by a very low sum of precipitation, was noted in Kętrzyn. In Suwałki this relation ratio was 6:52.

The $S P I$ is an index based on the probability of recording a given amount of precipitation, and the probabilities are normalised so that an index of zero indicates the median precipitation amount - half of the historical precipitation amounts are below the median, and half are above the median (Bąk, Łabędzki 2002). The index is negative for drought, and positive for wet conditions. As the dry or wet conditions become more severe, the index becomes more negative or positive. The SPI is computed for several time scales, ranging from one month to 24 months, to capture the various scales of both short-term and long-term drought (Dubrovsky et al. 2009).

Although the SPI was calculated for the months of the vegetation season for the whole three-decade period, Table 5 presents only a summary of these calculations as the basic statistics. According to this criterion, the deepest dry periods occurred in May regardless of the location, with the record-low value recorded in Mikołajki (-4.22). The maximum values of $S P I$ were reported in June and the absolute value for this month and the whole examined period, i.e. 2.55, was generated with a calculation for Elbląg in June. In general, the average SPI values for 1981-2010 were slightly below zero in May and June and slightly below zero for parts of August, September and October (in the latter case only in Elbląg). April and July were, on average, the months without symptoms of drought in all locations in the region. According to this indicator, there was a low variability in relation to the other parameters over the period of thirty years (the variability index: from 0.89 to 1.11 ). Higher values of variability were recorded in May and June and lower values were recorded in July. There was no statistically proven tendency of variation over time, as confirmed by the values of $\mathrm{R}^{2}$ and linear equations of the SPI index in time, presented in Table 5.

The Selianinov Index most comprehensively describes humidity conditions in relation to the needs of the environment. By using this criterion, the volume of precipitation is determined as well as the potential for its use by plants, depending on the thermal conditions. Table 6 includes only the average values for the decades and the total value for thirty years. From this perspective, actual situations in the individual months are not visible, but it can be concluded that the average values indicate a situation that reflects a good provision of water on the decadal scale. However, the numbers that are in each case higher than one were generated by averaging this index for the ranges from 0.3 to over 4 . Based on this, only one generalisation can be made - that the hydrothermal system was most beneficial over the three decades in August to September, whereas it was the worst from April to May.

In terms of its utility value for the environment and potential hazards, precipitation differs markedly. Low intensity of precipitation is associated with substantial water loss, especially at high temperatures, while high intensity is dangerous to cultivated plants, increases the risk of erosion or can cause flooding. The analysis of this situation was performed by determining the frequency of precipitation in different ranges of the daily sums (unfortunately, there is a lack of data that would allow for the estimation of the actual values of precipitation intensity per hour) and the results are compiled in Table 6. Precipitation sums in the range 5.1-10 mm were the most common. The occurrence of such a situation decreased from over $60 \%$, and in Mława from over $70 \%$, in the first and second decade to below $50 \%$ in the third decade. This tendency coincided with an increase in precipitation incidents in the range from 0.1 to $5 \mathrm{~mm}$ over a day. This tendency is confirmed by the results recorded for Poland (Łupikasza 2010). Here, a downward trend was found in the occurrence of days with high daily precipitation totals in Poland over the years 1951-2006. The frequencies in the ranges from 10.1 to 20 $\mathrm{mm}$ and from 20.1 to $30 \mathrm{~mm}$ also significantly decreased. Precipitation over $30 \mathrm{~mm}$ was extremely rare and no precipitation incidents below $1 \%$ were recorded. Cases of precipitation with the higher intensity over $70 \mathrm{~mm}$ per day were very rare. Such incidents did not occur in Mikołajki and there were up to $0.03 \%$ of such incidents in Elbląg (which indicates, in practice, a maximum of 2 such situations over thirty years recorded in the region of a given meteorological station). In general, almost two-thirds of the cases over thirty years were less intensive precipitation (not presenting a risk to the environment), which created good conditions for their practical usage. Extremely low precipitation (below $5 \mathrm{~mm}$ ) constituted slightly over $20 \%$ of the situations, and more intensive precipitation (from 10 to $20 \mathrm{~mm}$ ) occurred with a $10 \%$ frequency. There were no significant spatial differences in the region, apart from a slightly higher proportion of precipitation from the most 
Table 5. Standardized Precipitation Index $(S P I)$

\begin{tabular}{|c|c|c|c|c|c|c|c|}
\hline Values/Months & $\overline{\bar{z}}$ & 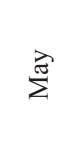 & 导 & 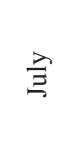 & 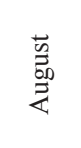 & 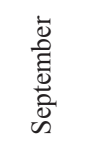 & $\begin{array}{l}\overrightarrow{\overline{0}} \\
\stackrel{0}{0} \\
0\end{array}$ \\
\hline \multicolumn{8}{|c|}{ Elbląg } \\
\hline Mean SPI & 0,04 & $-0,11$ & $-0,19$ & 0,07 & $-0,11$ & $-0,00$ & $-0,07$ \\
\hline Minimal value & $-2,13$ & $-2,49$ & $-1,99$ & $-1,50$ & $-1,79$ & $-2,46$ & $-2,34$ \\
\hline Maximal value & 2,05 & 2,19 & 2,55 & 2,33 & 1,67 & 1,87 & 1,45 \\
\hline Coefficient of variability & 0,98 & 1,01 & 1,07 & 0,87 & 0,98 & 1,08 & 1,03 \\
\hline $\mathrm{R}^{2}$ of trend & 0,00 & 0,09 & 0,00 & 0,02 & 0,02 & 0,07 & 0,12 \\
\hline \multicolumn{8}{|c|}{ Kętrzyn } \\
\hline Mean SPI & 0,13 & $-0,04$ & $-0,06$ & 0,00 & $-0,09$ & 0,07 & 0,10 \\
\hline Minimal value & $-1,96$ & $-3,84$ & $-1,83$ & $-1,74$ & $-2,42$ & $-2,47$ & $-2,29$ \\
\hline Maximal value & 2,21 & 1,52 & 2,28 & 2,10 & 1,78 & 1,74 & 1,73 \\
\hline Coefficient of variability & 0,98 & 1,02 & 1,02 & 0,93 & 0,98 & 1,06 & 0,93 \\
\hline $\mathrm{R}^{2}$ of trend & 0,04 & 0,00 & 0,01 & 0,00 & 0,03 & 0,07 & 0,07 \\
\hline \multicolumn{8}{|c|}{ Mikołajki } \\
\hline Mean SPI & 0,13 & $-0,01$ & $-0,06$ & 0,03 & $-0,09$ & 0,01 & 0,11 \\
\hline Minimal value & $-1,60$ & $-4,22$ & $-1,88$ & $-1,55$ & $-2,63$ & $-2,44$ & $-1,90$ \\
\hline Maximal value & 2,18 & 1,82 & 1,98 & 2,34 & 1,69 & 1,63 & 1,73 \\
\hline Coefficient of variability & 0,98 & 1,09 & 0,98 & 0,90 & 1,02 & 1,11 & 0,90 \\
\hline $\mathrm{R}^{2}$ of trend & 0,02 & 0,00 & 0,03 & 0,02 & 0,03 & 0,04 & 0,06 \\
\hline \multicolumn{8}{|c|}{ Mława } \\
\hline Mean SPI & 0,04 & $-0,07$ & $-0,07$ & 0,10 & $-0,03$ & 0,00 & 0,17 \\
\hline Minimal value & $-2,53$ & $-3,49$ & $-1,98$ & $-1,44$ & $-1,81$ & $-2,43$ & $-1,89$ \\
\hline Maximal value & 2,03 & 1,35 & 1,70 & 1,92 & 1,68 & 1,74 & 1,63 \\
\hline Coefficient of variability & 0,98 & 0,95 & 1,05 & 0,89 & 0,91 & 1,10 & 0,90 \\
\hline $\mathrm{R}^{2}$ of trend & 0,00 & 0,13 & 0,02 & 0,01 & 0,01 & 0,03 & 0,04 \\
\hline \multicolumn{8}{|c|}{ Olsztyn } \\
\hline Mean SPI & 0,07 & $-0,05$ & $-0,07$ & 0,10 & 0,03 & 0,08 & 0,12 \\
\hline Minimal value & $-2,11$ & $-3,62$ & $-1,85$ & $-1,64$ & $-1,79$ & $-2,34$ & $-2,09$ \\
\hline Maximal value & 2,30 & 1,30 & 2,05 & 2,24 & 1,74 & 1,60 & 1,41 \\
\hline Coefficient of variability & 1,01 & 0,97 & 0,97 & 0,92 & 1,00 & 1,08 & 0,96 \\
\hline $\mathrm{R}^{2}$ of trend & 0,00 & 0,03 & 0,00 & 0,00 & 0,03 & 0,10 & 0,08 \\
\hline \multicolumn{8}{|c|}{ Suwałki } \\
\hline Mean SPI & 0,18 & $-0,09$ & $-0,09$ & 0,01 & $-0,04$ & $-0,00$ & $-0,02$ \\
\hline Minimal value & $-2,26$ & $-2,88$ & $-1,87$ & $-2,13$ & $-1,94$ & $-2,26$ & $-2,62$ \\
\hline Maximal value & 2,22 & 1,82 & 2,20 & 2,15 & 1,92 & 1,61 & 1,59 \\
\hline Coefficient of variability & 0,94 & 1,04 & 0,97 & 0,91 & 0,91 & 1,04 & 0,91 \\
\hline $\mathrm{R}^{2}$ of trend & 0,04 & 0,00 & 0,02 & 0,00 & 0,05 & 0,11 & 0,07 \\
\hline
\end{tabular}

common range. The total number of days with precipitation in relation to the total number of days in the three decades was $66 \%$ in the whole region.

Full comprehensive assessment of moisture conditions is possible only after taking into account the transpiration field (Bac, Kuchar 2001; Bac et al. 2008; Dubrovsky et al. 2009; Orlińska-Woźniak et al. 2013). Unfortunately, there is sufficient data for the calculation of this type in all locations.

Rainfalls with thunder are a separate category of precipitation, as it is characterised by high intensity combined with fierce winds. The highest daily precipitation totals recorded in Poland are formed in connection with the activity of Mediterranean cyclones, especially in summer (Degirmendžić, Kożuchowski 2015). Rainfalls with thunder can therefore be regarded as a class of extreme climatic events. In many cases, these situations are detri- mental to the environment as they are destructive to vegetating plants (e.g. they cause cereal and rape lodging). In the analysed region, thunder occurred throughout the year, but its nature and number was much lower in winter and autumn, and spring. It was found that the number of thunder events decreased in the over the decades of the thirty-year period. In 1981-1990 and 1991-2000, the number of thunder storms was twice as high as in 2001-2010. On average, there were slightly more thunder storms in Elbląg and Mikołajki, with the lowest number recorded in Kętrzyn. In the annual arrangement, the period from May to August is thought to be a typically thunder storm season when from 4 to 7 incidents were recorded on average in the first two decades, whereas this number amounted to 3 to 5 in the last decade. It is very probably that the circulation situation had an impact on precipitation (BielecBąkowska 2003). 
Table 6. Selianinov index in years 1981-2010

\begin{tabular}{|c|c|c|c|c|c|c|c|c|}
\hline $\begin{array}{l}\text { Weather stations/ } \\
\text { Months }\end{array}$ & $\overline{\bar{z}}$ & $\sum^{\vec{E}}$ & $\stackrel{\Xi}{\Xi}$ & $\vec{\partial}$ & $\begin{array}{l}\vec{w} \\
\stackrel{\overrightarrow{0}}{00} \\
\vec{Z}\end{array}$ & 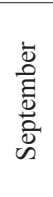 & $\begin{array}{l}\dot{0} \\
\stackrel{0}{0} \\
\dot{0} \\
0\end{array}$ & $\begin{array}{l}\dot{0} \\
\frac{0}{0} \\
\frac{0}{0} \\
0 \\
1 \\
\frac{1}{0} \\
\overline{0}\end{array}$ \\
\hline \multicolumn{9}{|c|}{ Years 1981-1990 } \\
\hline Elbląg & 1,6 & 1,1 & 2,3 & 1,4 & 1,5 & 2 & 1,7 & 1,6 \\
\hline Kętrzyn & 1,5 & 1,3 & 2 & 1,5 & 1,3 & 1,6 & 1,4 & 1,5 \\
\hline Mikołajki & 1,3 & 1,4 & 2,2 & 1,3 & 1,5 & 1,5 & 1,2 & 1,5 \\
\hline Mława & 1,3 & 1 & 1,8 & 1,3 & 1,1 & 1,3 & 1,2 & 1,3 \\
\hline Olsztyn & 1,4 & 1,2 & 2,1 & 1,3 & 1,2 & 1,7 & 1,5 & 1,5 \\
\hline Suwałki & 1,6 & 1,5 & 2,1 & 1,5 & 1,3 & 1,8 & 1,8 & 1,7 \\
\hline \multicolumn{9}{|c|}{ Years 1991-2000 } \\
\hline Elbląg & 1,6 & 1,7 & 1,5 & 1,2 & 1,5 & 2,3 & 2,9 & 1,8 \\
\hline Kętrzyn & 2,2 & 1,5 & 1,7 & 1,2 & 1,2 & 1,6 & 2 & 1,6 \\
\hline Mikołajki & 2,1 & 1,5 & 1,5 & 1,3 & 1,3 & 1,6 & 2,1 & 1,6 \\
\hline Mława & 2 & 1,4 & 1,5 & 1,3 & 0,9 & 2 & 1,8 & 1,5 \\
\hline Olsztyn & 2,3 & 1,6 & 1,5 & 1,4 & 1 & 1,7 & 2,2 & 1,7 \\
\hline Suwałki & 2,3 & 1,3 & 1,4 & 1,4 & 1,1 & 1,6 & 2,5 & 1,6 \\
\hline \multicolumn{9}{|c|}{ Years 2000-2010 } \\
\hline Elbląg & 1,5 & 1,6 & 1,5 & 1,6 & 1,5 & 1,3 & 3,3 & 1,7 \\
\hline Kętrzyn & 1,2 & 1,5 & 1,5 & 1,4 & 1,7 & 1,4 & 2,6 & 1,6 \\
\hline Mikołajki & 1,2 & 1,6 & 1,6 & 1,3 & 1,8 & 1,4 & 2,6 & 1,6 \\
\hline Mława & 1,1 & 1,7 & 1,3 & 1,3 & 1,2 & 1,1 & 2 & 1,4 \\
\hline Olsztyn & 1,3 & 1,6 & 1,7 & 1,5 & 1,5 & 1,3 & 2,8 & 1,6 \\
\hline Suwałki & 1,1 & 1,7 & 1,5 & 1,6 & 1,6 & 1,3 & 3,1 & 1,7 \\
\hline \multicolumn{9}{|c|}{ Years 1981-2010 } \\
\hline Elbląg & 1,6 & 1,4 & 1,8 & 1,4 & 1,5 & 1,9 & 2,6 & 1,7 \\
\hline Kętrzyn & 1,6 & 1,4 & 1,7 & 1,4 & 1,4 & 1,5 & 2 & 1,6 \\
\hline Mikołajki & 1,5 & 1,5 & 1,8 & 1,3 & 1,6 & 1,5 & 2 & 1,6 \\
\hline Mława & 1,5 & 1,4 & 1,5 & 1,3 & 1,1 & 1,4 & 1,6 & 1,4 \\
\hline Olsztyn & 1,7 & 1,5 & 1,8 & 1,4 & 1,2 & 1,6 & 2,2 & 1,6 \\
\hline Suwałki & 1,7 & 1,5 & 1,6 & 1,5 & 1,3 & 1,6 & 2,5 & 1,7 \\
\hline
\end{tabular}

The data presented above, accompanied with the analyses, describes how, on a very small scale, it is not possible to provide a clear answer to the fundamental question about precipitation in the future. However, some indicators of rain tendency seem to fit well with the trends described by those authors who have dealt with the problem of future precipitation across Europe. It is proven that precipitation trends since 1960 show an increase by up to $70 \mathrm{~mm}$ per decade in north-eastern and north-western Europe, in particular in winter, and a decrease by up to $90 \mathrm{~mm}$ per decade in some parts of southern Europe, in particular in summer (Klein et al. 2002). Some of our results also agree with the results found by Casanueva et al. (2014): in winter, both the mean precipitation and consecutive wet days present an upwards (downwards) trend in the north (south) of the continent, while in summer these two variables decrease (increase) in the west (east) of Europe. A general assumption is that the summer precipitation all over Central Europe (except along the coast of the Baltic Sea - see data about future tendencies from Elblag) will decrease, while in most cases Central Europe will most likely become wetter in the winter season. Despite these precipitation increases, the amount of snow and the area covered by snow are expected to decline due to warming (Anders et al. 2014).

\section{Conclusions}

- The spatial distribution of precipitation in the region indicated that the highest average annual values in the period of 1981-2010 (700.1 mm) were recorded in Elbląg whereas the lowest $(555.3 \mathrm{~mm})$ were noted in Mława; this proves a decrease in the precipitation sums from the north-western part of the region towards the east and south-east.

- The highest precipitation sums occurred in the last decade, namely, in 2001-2010. Despite this, the statistical analysis did not demonstrate any significant tendency of precipitation change over thirty years.

- The summer precipitations (from June to August) were predominant in the whole region, while the lowest precipitations were recorded from December to April. On a monthly scale, there are some cases of statistically significant tendencies towards an increase in precipi- 
Table 7. Frequency of daily sums of precipitation in intervals as percentages of total days with precipitation - in years 1981-2010

\begin{tabular}{|c|c|c|c|c|}
\hline $\begin{array}{c}\text { Intervals of sums } \\
\text { of precipitation }\end{array}$ & $1981-1990$ & $1991-2000$ & 2001-2010 & $\begin{array}{c}\text { Average: } \\
1981-2010\end{array}$ \\
\hline \multicolumn{5}{|c|}{ Elbląg } \\
\hline$>70 \mathrm{~mm}$ & 0,00 & 0,04 & 0,03 & 0,03 \\
\hline $60,1-70 \mathrm{~mm}$ & 0,05 & 0,00 & 0,00 & 0,02 \\
\hline $50,1-60 \mathrm{~mm}$ & 0,19 & 0,04 & 0,07 & 0,10 \\
\hline $40,1-50 \mathrm{~mm}$ & 0,38 & 0,40 & 0,10 & 0,29 \\
\hline $30,1-40 \mathrm{~mm}$ & 1,03 & 0,76 & 0,71 & 0,83 \\
\hline $20,1-30 \mathrm{~mm}$ & 6,01 & 6,16 & 5,53 & 5,90 \\
\hline $10,1-20 \mathrm{~mm}$ & 12,73 & 12,04 & 9,13 & 11,30 \\
\hline $5,1-10 \mathrm{~mm}$ & 61,25 & 62,40 & 48,17 & 57,27 \\
\hline $0,1-5 \mathrm{~mm}$ & 18,37 & 18,15 & 36,25 & 24,26 \\
\hline \multicolumn{5}{|c|}{ Kętrzyn } \\
\hline$>70 \mathrm{~mm}$ & 0,00 & 0,00 & 0,03 & 0,01 \\
\hline $60,1-70 \mathrm{~mm}$ & 0,04 & 0,00 & 0,10 & 0,05 \\
\hline $50,1-60 \mathrm{~mm}$ & 0,04 & 0,00 & 0,03 & 0,03 \\
\hline $40,1-50 \mathrm{~mm}$ & 0,27 & 0,28 & 0,10 & 0,22 \\
\hline $30,1-40 \mathrm{~mm}$ & 1,03 & 0,78 & 0,49 & 0,77 \\
\hline $20,1-30 \mathrm{~mm}$ & 4,08 & 4,79 & 4,30 & 4,39 \\
\hline $10,1-20 \mathrm{~mm}$ & 10,95 & 10,60 & 8,18 & 9,91 \\
\hline $5,1-10 \mathrm{~mm}$ & 63,15 & 63,58 & 47,64 & 58,12 \\
\hline $0,1-5 \mathrm{~mm}$ & 20,42 & 19,96 & 39,10 & 26,50 \\
\hline \multicolumn{5}{|c|}{ Mikołajki } \\
\hline$>70 \mathrm{~mm}$ & 0,00 & 0,00 & 0,00 & 0,00 \\
\hline $60,1-70 \mathrm{~mm}$ & 0,13 & 0,00 & 0,03 & 0,05 \\
\hline $50,1-60 \mathrm{~mm}$ & 0,09 & 0,13 & 0,14 & 0,12 \\
\hline $40,1-50 \mathrm{~mm}$ & 0,30 & 0,04 & 0,31 & 0,22 \\
\hline $30,1-40 \mathrm{~mm}$ & 0,85 & 1,07 & 1,05 & 0,99 \\
\hline $20,1-30 \mathrm{~mm}$ & 3,80 & 4,50 & 3,36 & 3,89 \\
\hline $10,1-20 \mathrm{~mm}$ & 9,65 & 10,17 & 7,42 & 9,08 \\
\hline $5,1-10 \mathrm{~mm}$ & 60,46 & 58,82 & 46,75 & 55,34 \\
\hline $0,1-5 \mathrm{~mm}$ & 24,72 & 25,27 & 40,95 & 30,31 \\
\hline \multicolumn{5}{|c|}{ Mława } \\
\hline$>70 \mathrm{~mm}$ & 0,05 & 0,00 & 0,00 & 0,02 \\
\hline $60,1-70 \mathrm{~mm}$ & 0,00 & 0,10 & 0,03 & 0,04 \\
\hline $50,1-60 \mathrm{~mm}$ & 0,05 & 0,05 & 0,07 & 0,06 \\
\hline $40,1-50 \mathrm{~mm}$ & 0,25 & 0,39 & 0,03 & 0,23 \\
\hline $30,1-40 \mathrm{~mm}$ & 0,91 & 0,98 & 0,62 & 0,84 \\
\hline $20,1-30 \mathrm{~mm}$ & 4,10 & 4,38 & 3,73 & 4,07 \\
\hline $10,1-20 \mathrm{~mm}$ & 9,41 & 9,98 & 7,98 & 9,12 \\
\hline $5,1-10 \mathrm{~mm}$ & 71,57 & 70,50 & 46,27 & 62,78 \\
\hline $0,1-5 \mathrm{~mm}$ & 13,66 & 13,62 & 41,26 & 22,84 \\
\hline \multicolumn{5}{|c|}{ Olsztyn } \\
\hline$>70 \mathrm{~mm}$ & 0,00 & 0,00 & 0,03 & 0,01 \\
\hline $60,1-70 \mathrm{~mm}$ & 0,05 & 0,00 & 0,03 & 0,03 \\
\hline $50,1-60 \mathrm{~mm}$ & 0,14 & 0,00 & 0,03 & 0,06 \\
\hline $40,1-50 \mathrm{~mm}$ & 0,23 & 0,13 & 0,24 & 0,20 \\
\hline $30,1-40 \mathrm{~mm}$ & 0,87 & 1,12 & 0,85 & 0,95 \\
\hline $20,1-30 \mathrm{~mm}$ & 4,37 & 5,22 & 3,67 & 4,42 \\
\hline $10,1-20 \mathrm{~mm}$ & 12,02 & 10,79 & 8,81 & 10,54 \\
\hline $5,1-10 \mathrm{~mm}$ & 62,34 & 63,49 & 46,26 & 57,36 \\
\hline $0,1-5 \mathrm{~mm}$ & 19,99 & 19,24 & 40,07 & 26,43 \\
\hline \multicolumn{5}{|c|}{ Suwałki } \\
\hline$>70 \mathrm{~mm}$ & 0,00 & 0,00 & 0,07 & 0,02 \\
\hline $60,1-70 \mathrm{~mm}$ & 0,05 & 0,05 & 0,04 & 0,04 \\
\hline $50,1-60 \mathrm{~mm}$ & 0,14 & 0,05 & 0,04 & 0,07 \\
\hline $40,1-50 \mathrm{~mm}$ & 0,09 & 0,10 & 0,14 & 0,11 \\
\hline $30,1-40 \mathrm{~mm}$ & 1,25 & 0,67 & 0,84 & 0,92 \\
\hline $20,1-30 \mathrm{~mm}$ & 4,49 & 5,09 & 3,75 & 4,44 \\
\hline $10,1-20 \mathrm{~mm}$ & 10,31 & 11,71 & 8,51 & 10,18 \\
\hline $5,1-10 \mathrm{~mm}$ & 66,42 & 64,73 & 47,36 & 59,50 \\
\hline $0,1-5 \mathrm{~mm}$ & 17,25 & 17,61 & 39,27 & 24,71 \\
\hline
\end{tabular}


Table 8. Occurrence of thunder storms in years 1981-2010

\begin{tabular}{|c|c|c|c|c|c|c|c|c|c|c|c|c|c|}
\hline $\begin{array}{l}\text { Weather stations/ } \\
\text { Months }\end{array}$ & 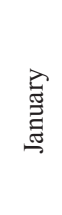 & 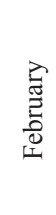 & $\begin{array}{l}\text { 总 } \\
\sum^{\pi}\end{array}$ & $\overline{\bar{z}}$ & $\stackrel{\vec{\Sigma}}{\Sigma}$ & 导 & $\stackrel{\gtrsim}{\Xi}$ & $\begin{array}{l}\vec{w} \\
\stackrel{\vec{\partial}}{0} \\
\vec{z}\end{array}$ & 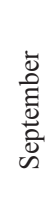 & $\begin{array}{l}\overline{0} \\
\overline{0} \\
\overline{0} \\
0\end{array}$ & $\begin{array}{l}\dot{\vec{D}} \\
\vec{\Xi} \\
\text { ठे } \\
z\end{array}$ & $\begin{array}{l}\bar{\Xi} \\
\text { हूँ } \\
\text { ڤ. }\end{array}$ & 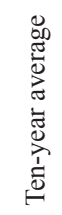 \\
\hline \multicolumn{14}{|c|}{ Years 1981-1990 } \\
\hline Elbląg & 0,1 & 0,0 & 0,0 & 1,9 & 4,3 & 5,8 & 4,2 & 3,6 & 1,3 & 0,4 & 0,2 & 0,1 & 21,9 \\
\hline Kętrzyn & 0,1 & 0,0 & 0,1 & 1,7 & 5,5 & 6,9 & 5,9 & 4,6 & 1,9 & 0,4 & 0,2 & 0,0 & 27,3 \\
\hline Mikołajki & 0,1 & 0,0 & 0,3 & 1,5 & 5,9 & 7,2 & 4,8 & 4,2 & 1,3 & 0,2 & 0,2 & 0,0 & 25,7 \\
\hline Mława & 0,0 & 0,0 & 0,3 & 1,9 & 4,3 & 4,7 & 4,5 & 3,4 & 1,0 & 0,3 & 0,0 & 0,0 & 20,4 \\
\hline Olsztyn & 0,5 & 0,0 & 0,9 & 2,1 & 5,0 & 5,2 & 4,1 & 3,0 & 0,8 & 0,5 & 0,1 & 0,3 & 22,5 \\
\hline Suwałki & 0,0 & 0,0 & 0,2 & 0,9 & 4,5 & 6,6 & 5,5 & 2,7 & 1,6 & 0,0 & 0,1 & 0,0 & 22,1 \\
\hline \multicolumn{14}{|c|}{ Years 1991-2000 } \\
\hline Elbląg & 0,0 & 0,1 & 0,3 & 1,1 & 4,8 & 5,8 & 5,1 & 6,5 & 2,2 & 0,6 & 0,1 & 0,1 & 26,7 \\
\hline Kętrzyn & 0,0 & 0,0 & 0,2 & 0,4 & 2,2 & 3,6 & 3,3 & 3,6 & 1,4 & 0,1 & 0,0 & 0,0 & 14,8 \\
\hline Mikołajki & 0,0 & 0,0 & 0,1 & 0,6 & 4,9 & 6,3 & 5,6 & 5,8 & 1,6 & 0,4 & 0,0 & 0,0 & 25,3 \\
\hline Mława & 0,0 & 0,0 & 0,0 & 1,6 & 5,0 & 4,7 & 5,5 & 4,3 & 1,7 & 0,4 & 0,0 & 0,0 & 23,2 \\
\hline Olsztyn & 0,0 & 0,1 & 0,5 & 1,3 & 5,2 & 4,7 & 4,0 & 4,1 & 0,8 & 0,2 & 0,1 & 0,1 & 21,1 \\
\hline Suwałki & 0,0 & 0,0 & 0,1 & 0,7 & 4,0 & 5,0 & 4,7 & 4,3 & 1,0 & 0,3 & 0,0 & 0,0 & 20,1 \\
\hline \multicolumn{14}{|c|}{ Years 2000-2010 } \\
\hline Elbląg & 0,0 & 0,1 & 0,1 & 0,5 & 1,9 & 2,5 & 4,5 & 1,7 & 1,3 & 0,2 & 0,0 & 0,0 & 12,8 \\
\hline Kętrzyn & 0,1 & 0,1 & 0,2 & 0,4 & 1,5 & 2,4 & 3,8 & 1,3 & 0,9 & 0,2 & 0,0 & 0,0 & 10,9 \\
\hline Mikołajki & 0,0 & 0,0 & 0,2 & 0,4 & 1,7 & 2,0 & 3,9 & 2,2 & 1,0 & 0,2 & 0,1 & 0,0 & 11,7 \\
\hline Mława & 0,0 & 0,0 & 0,1 & 0,8 & 2,2 & 2,2 & 4,3 & 2,0 & 1,3 & 0,1 & 0,0 & 0,0 & 13,0 \\
\hline Olsztyn & 0,2 & 0,3 & 0,3 & 0,5 & 1,7 & 2,0 & 3,8 & 1,8 & 1,0 & 0,2 & 0,0 & 0,0 & 11,8 \\
\hline Suwałki & 0,0 & 0,1 & 0,2 & 0,3 & 1,7 & 1,5 & 3,7 & 1,4 & 0,7 & 0,2 & 0,0 & 0,0 & 9,8 \\
\hline \multicolumn{14}{|c|}{ Years $1981-2010$} \\
\hline Elbląg & 0,0 & 0,1 & 0,1 & 1,2 & 3,7 & 4,7 & 4,6 & 3,9 & 1,6 & 0,4 & 0,1 & 0,1 & 20,5 \\
\hline Kętrzyn & 0,1 & 0,0 & 0,2 & 0,8 & 3,1 & 4,3 & 4,3 & 3,2 & 1,4 & 0,2 & 0,1 & 0,0 & 17,7 \\
\hline Mikołajki & 0,0 & 0,0 & 0,2 & 0,8 & 4,2 & 5,2 & 4,8 & 4,1 & 1,3 & 0,3 & 0,1 & 0,0 & 20,9 \\
\hline Mława & 0,0 & 0,0 & 0,1 & 1,4 & 3,8 & 3,9 & 4,8 & 3,2 & 1,3 & 0,3 & 0,0 & 0,0 & 18,9 \\
\hline Olsztyn & 0,2 & 0,1 & 0,6 & 1,3 & 4,0 & 4,0 & 4,0 & 3,0 & 0,9 & 0,3 & 0,1 & 0,1 & 18,5 \\
\hline Suwałki & 0,0 & 0,0 & 0,2 & 0,6 & 3,4 & 4,4 & 4,6 & 2,8 & 1,1 & 0,2 & 0,0 & 0,0 & 17,3 \\
\hline
\end{tabular}

tation in years 1981-2010, in winter, especially in February.

- The number of years with sums of precipitation below $75 \%$ of the multi-annual norm was very low, however the multi-annual means of precipitation for specific months in some cases were below $25 \%$ of the norm, but in some cases exceeded $00 \%$.

- The frequency of dry spells lasting for over 10 days ranged from 50 to 59 incidents over the thirty years. The longest average period without precipitation in the region was recorded in Suwałki (15.3 days) whereas the shortest was in Mikołajki (14.0 days).

- The average SPI values for 1981-2010 were slightly negative in May and June and partly slightly below zero in August, September, and October (in the latter case only in Elbląg). According to the average SPI index for the multi-annual period, April and July were the months without drought symptoms in all locations in the region.

- According to the Selianinov Index, the hydrothermal system was most beneficial between August and September over thirty years, whereas it was the worst from April till May.
- The intensity of daily precipitation was moderate and in general stayed within the $5.1-10 \mathrm{~mm}$ range. The most intense rainfalls with thunder were visibly reduced in the last decade of the analysed period.

Bibliography

Anders I., Stagl J., Auer I., Pavlik D., 2014, Climate change in central and eastern Europe, [in:] Managing protected areas in central and eastern Europe under climate change, Advances in Global Change Research, 58, Springer Netherlands, 17-30

Arnell N.W., 2004, Climate change and global water resources: SRES emissions and socio-economic scenarios, Global Environmental Change, 14 (1), 31-52, DOI: 10.1016/j.gloenvcha.2003.10.006

Bac S., Kuchar L., 2001, Modification of formula for evaluation of potential evaporation according to Turca, (in Polish), Annales Universitatis Mariae Curie-Skłodowska, Section B: Geographia, Geologia, Mineralogia et Petrographia, 55/56, 41-49

Bac S., Iwański S., Kuchar L., 2008, Estimation of potential evaporation for the winter season for the needs of hydrological modeling, (in Polish), Acta Agrophisica, 12 (2), 305-314 
Banaszkiewicz B., Dragańska E., Szwejkowski Z., 2004a, Characteristics of selected moisture and precipitation conditions in north-eastern Poland in years 1971-2000, (in Polish), [in:] Bilanse wodne ekosystemów rolniczych, M. Rojek (ed.), Seria Monografie AR Wrocław, XXXVIII, 503, 23-30

Banaszkiewicz B., Grabowska K., Szwejkowski. Z., 2004b, Characteristics of precipitations on the area of Warmia and Mazury Voivodship in years 2000-2002, (in Polish), Acta Agrophisica, 3 (1), 5-11

Banaszkiewicz B., Grabowska K., Szwejkowski Z., Grabowski J., 2006, Characteristics of precipitation condition in Bałcyny and Tomaszkowo in 1996-2005, (in Polish), Przegląd Naukowy Inżynieria i Kształtowanie Środowiska, 1 (33), 137-145

Bąk B., Łabędzki L., 2002, Assessing drought severity with the relative precipitation index $(R P I)$ and the standardized precipitation index $(S P I)$, Journal of Water and Land Development, 12 (6), 89-105

Bielec-Bąkowska Z., 2003, Long-term variability of thunderstorm occurrence in Poland in the $20^{\text {th }}$ century, Atmospheric Research, 67-68, 35-52, DOI: 10.1016/S0169-8095(03)00082-6

Casanueva A., Rodríguez-Puebla C., Frías M.D., González-Reviriego N., 2014, Variability of extreme precipitation over Europe and its relationships with teleconnection patterns, Hydrology and Earth System Sciences, 18, 709-725, DOI: 10.5194/hess-18-709-2014

Degirmendžić J., Kożuchowski K., 2015, Precipitation of the Mediterranean origin in Poland - its seasonal and long-term variability, Quaestiones Geographicae, 34 (1), 37-53, DOI: 10.1515/quageo-2015-0004

Dragańska E., Szwejkowski Z., Banaszkiewicz B., 2005, Excesses and shortages of rainfall during the growing season in north-eastern Poland in years 1971-2000, (in Polish), WodaŚrodowisko-Obszary Wiejskie, 5 (14), 119-128

Dubrovsky M., Svoboda M.D, Trnka M., Hayes M.J., Wilhite D.A., Zalud Z., Hlavinka P., 2009, Application of relative drought indices in assessing climate-change impacts on drought conditions in Czechia, Theoretical and Applied Climatology, 96 (1), 155-171, DOI: 10.1007/s00704-008-0020-x

Grabowska K., Banaszkiewicz B., Szwejkowski Z., 2004, Shortages and excess rainfall weather in Warmia-Mazury voivodship in the years 2000-2002, (in Polish), Acta Agrophisica, 3 (1), 57-64

ISOK, 2014, Maps of flood risk in Poland, (in Polish), KZGW, available at http://mapy.isok.gov.pl/imap/ (data access 22.11.2016)

Klein Tank A.M.G., Wijngaard J. B., Können G.P., Böhm R., Demarée G., Gocheva A., Mileta M., Pashiardis S., Hejkrlik
L., Kern-Hansen C., Heino R., Bessemoulin P., Müller-Westermeier G., Tzanakou M., Szalai S., Pálsdóttir T., Fitzgerald D., Rubin S., Capaldo M., Maugeri M., Leitass A., Bukantis A., Aberfeld R., van Engelen A.F.V., Forland E., Miętus M., Coelho F., Mares C., Razuvaev V., Nieplova E., Cegnar T., Antonio López J., Dahlström B., Moberg A., Kirchhofer W., Ceylan A., Pachaliuk O., Alexander L.V., Petrovic P., 2002, Daily dataset of 20th-century surface air temperature and precipitation series for the European Climate Assessment, International Journal of Climatology, 22 (12), 1441-1453, DOI: $10.1002 /$ joc. 773

Kondracki J., 1994, Geography of Poland. Physico-geographical Mesoregions, (in Polish), PWN, Warszawa, 240 pp.

Kuchar L., Otop I.,, 2004, Variability of precipitation during the summer season in Jelenia Gora in years 1951-2000, (in Polish), Acta Scientiarum Polonorum. Formatio Circumiectus, 3 (2), 101-111

Lehner B., Döll P., Alcamo J., Henrichs T., Kaspar F., 2006, Estimating the impact of global change on flood and drought risks in Europe: a continental, integrated analysis, Climatic Change, 75 (3), 273-299, DOI: 10.1007/s10584-006-6338-4

Łupikasza E., 2010, Relationships between occurrence of high precipitation and atmospheric circulation in Poland using different classifications of circulation types, Physics and Chemistry of the Earth. Parts A/B/C, 35 (9-12), 448-455, DOI: 10.1016/j.pce.2009.11.012

Orlińska-Woźniak P., Wilk P., Gębala J., 2013, Water availability in reference to water needs in Poland, Meteorology Hydrology and Water Management, 1 (1), 45-50

Przedpełska W., 1971, Atmospheric droughts in Poland and method its description, (in Polish), Prace PIHM, 103, 3-27

Suchecki S., Szwejkowski Z., Dragańska E., Olba-Zięty E., 2011, Thermal and pluviometric characteristics of north-eastern Poland in years 1966-2005, [in] Contemporary Problems of Management and Environmental Protection, 10, 7-68

Szalińska W., Otop I., Tokarczyk T., 2014, Precipitation extremes during flooding in the Odra River basin in May-June 2010, Meteorology Hydrology and Water Management, 2 (1), 13-20

Szwejkowski Z., Dragańska E., Banaszkiewicz B., 2005, Precipitations in northern-eastern Poland in years 1971-2000, (in Polish), Zeszyty Problemowe Postępów Nauk Rolniczych, $505,431-437$

Szwejkowski Z., Nowicka A., Dragańska E., 2002, Climate of Mazury Lake District. Part I: Temperature and precipitation in years 1951-1995, (in Polish), Fragmenta Agronomica, 2 (74), 285-296 